\title{
Pricing and Hedging American Options Analytically: A Perturbation Method
}

\author{
Jin E. Zhang ${ }^{1}$ \\ School of Economics and Finance \\ The University of Hong Kong \\ Pokfulam Road, Hong Kong \\ Email: jinzhang@hku.hk \\ Tiecheng Li \\ Department of Mathematical Science \\ Tsinghua University \\ Beijing 100084, P.R. China \\ Email: tli@math.tsinghua.edu.cn
}

First Version: March 2003

Final Version: July 2009

Forthcoming in Mathematical Finance

Keywords: Analytical formula; Critical stock price; American option; Black-Scholes equation; Perturbation method

JEL Classification Code: G13

\footnotetext{
${ }^{1}$ Corresponding author. Tel: (852) 2859 1033, Fax: (852) 2548 1152. The authors acknowledge Dilip Madan (managing editor) and Sateesh Mane (referee) for helpful comments and suggestions, and Min Dai for assistance in numerical computations. Jin E. Zhang appreciates having interesting discussions with Yacine Aït-Sahalia, Peter Bossaerts, Peter Carr, Herb Johnson, Nengjiu Ju, Marti Subrahmanyam, Theodore Y. Wu and Chu Zhang. Tiecheng Li has benefited from interesting discussions with Lishang Jiang and Fahuai Yi. Jin E. Zhang has been supported by grants from the Research Grants Council of the Hong Kong Special Administrative Region, China (Project No. HKU 7427/06H and HKU 7549/09H).
} 


\title{
Pricing and Hedging American Options Analytically: A Perturbation Method
}

\begin{abstract}
This paper studies the critical stock price of American options with continuous dividend yield. We solve the integral equation and derive a new analytical formula in a series form for the critical stock price. American options can be priced and hedged analytically with the help of our critical-stock-price formula. Numerical tests show that our formula gives very accurate prices. With the error well controlled, our formula is now ready for traders to use in pricing and hedging the S\&P 100 index options and for the Chicago Board Options Exchange to use in computing the VXO volatility index.
\end{abstract}




\section{Introduction}

American options currently trade throughout the world. The most popular American option contracts in the United States are those on the S\&P 100 Index (OEX), traded on the Chicago Board Options Exchange (CBOE). For example, the open interest of S\&P 100 Index options on March 12, 2003 was 325,810 of which 167,768 contracts were calls and 157,042 contracts were puts. The trading volume was 72,713 , including 37,703 calls and 35,010 puts. Most foreign-currency options traded on the Philadelphia Stock Exchange (PSE) are American style. For example, the total open interest of foreign-currency options on March 11, 2003 was 15,616 of which 12,702 contracts were American options.

Given the fact that American options are frequently traded on exchanges, pricing American options is very important. Due to the difficulty of dealing with the early-exercise feature, a closed-form formula ${ }^{2}$ has not been found, and it seems unlikely that one will be found any time soon. In practice, the price of American options is often computed numerically by Cox, Ross and Rubinstein's (1979) binomial-tree method, by solving Black-Scholes (1973) partial differential equation with a moving boundary, or by solving an integral equation for the critical stock price ${ }^{3}$ (see, e.g., Yu (1993), and Huang, Subrahmanyam and Yu (1996)). Even though these numerical methods are able to give accurate values, a good analytical approximate formula is still very useful and valuable for four reasons. First, the numerical computation could be time consuming. Second, an analytical formula can be used in case a computing engine is not available. Third, the detailed study of the critical stock price provides a methodology to study other moving boundary problems in finance, such as convertible bonds and real options with an early-exercise feature. Fourth, an analytical formula provides intuition of the relation between parameters. We focus on

\footnotetext{
${ }^{2}$ In this paper, we differentiate between two concepts: a closed-form formula and an analytical formula. By a closed-form formula, we mean that the formula is written in an easily computable function such as power, exponential or logarithmical functions, or a special function such as cumulative normal distribution, Bessel or confluent hypergeometric functions, etc. But the concept of an analytical formula has a wider scope. It covers that of a closed-form formula and a summation or multiplication series of some known functions.

${ }^{3}$ The critical stock price, the early-exercise boundary and the optimal exercise boundary have the same meaning. These terms are used interchangeably in this paper.
} 
analytical approaches in this paper.

Johnson (1983) proposes an analytical approximation for the American put price based on a regression on Parkinson's (1977) numerical values. The formula is generated based on numbers through a statistical method instead of on a rigorous analysis of the intrinsic nature of the problem. Geske and Johnson (1984) give an analytical expression by treating an American put as a portfolio of an infinite number of compound options. Evaluating the multivariate cumulative normal distribution function is a practical problem for this method. They propose using a four-point extrapolation method to evaluate American options approximately. MacMillan (1986) and Barone-Adesi and Whaley (1987) use quadratic approximation for American option prices and find the critical stock price numerically by iteration. Bunch and Johnson (1992) propose a modified two-point Geske-Johnson method. Broadie and Detemple (1996) derive lower and upper bounds for the American option price. A comparison of the different methods is available in Broadie and Detemple (1996) and Ju $(1998)^{4}$.

It is by now well known that the price of American options can be written as the sum of the corresponding European option price and an integral in terms of its early-exercise boundary. The mathematical result appeared early in the literature by Kolodner (1956) and McKean (1965). It has been restudied by Kim (1990), Jacka (1991), and Carr, Jarrow and Myneni (1992) to gain economic insights. The financial problem of pricing American options boils down to a mathematical problem of solving an integral equation with the critical stock price as an unknown function of time and other parameters. Ju (1998) approximates the early-exercise boundary as a piece-wise exponential function, obtains an analytical formula for the American option price, and then uses numerical iteration to determine a more accurate boundary. An analytical formula for the critical stock price has not yet been found. Even for the leading-order expansion near expiration, Kuske and Keller (1998), Stamicar, Ševčovič and Chadam (1999), and Bunch and Johnson (2000) give

\footnotetext{
${ }^{4}$ American option pricing has also been studied numerically by Brennan and Schwartz (1977) with a finite-difference method, by Carr (1998) with a randomization technique, and by Longstaff and Schwartz (2001) with a simulation-based least-squares approach.
} 
different analytical expressions. In a recent development, Chen and Chadam (2007) provide a convincing mathematical justification to show that Ševčovič and Chadam (1999) give the correct asymptotic behavior near expiration, which is also confirmed by Evans, Kuske and Keller (2002). But Chen and Chadam (2007) only study the case when the underlying asset does not pay any dividend. We nontrivially extend Chen and Chadam's (2007) methodology to the case with dividend yield that models the price of American options on stock indices and currencies, which is one of the central concerns of academics and market participants. Recently Zhu (2006) develops a quasi-analytical expression for the critical stock price, however his complicated iteration procedure requires numerical integration in each step. An accurate and user-friendly approximate formula for the critical stock price is not available.

This paper makes three contributions to the literature. The first contribution is to entend the Chen and Chadam (2007) approach by taking into account a dividend yield. The second contribution is to derive approximate solutions for the critical stock price of the American option with continuous dividend yield at small $\sigma^{2} T$, for three different regions where the difference between interest rate and dividend yield is positive, zero and negative. With the help of the critical-stock-price formula, one can price and hedge American options analytically. The third contribution is to test the validity of the formula. Compared with the highly accurate numerical values computed by solving the integral equation, our formulas up to the fourth-order term give very accurate prices with an accuracy up to 0.01 cent for the American options, with a one-month maturity and a strike price of 100 dollars, tested in this paper. With the error well controlled, our formula is now ready for traders to use in pricing OEX options, since most liquid OEX options have a maturity of about a month. The formula can also be used by the CBOE to compute the VXO volatility index since the index is defined as an implied volatility of the OEX options with one-month maturity. 


\section{The Model}

For completeness, this section briefly reviews the Black-Merton-Scholes (1973) model of American option pricing. In a risk-neutral world, the price of an underlying stock, $S_{t}$, is modelled by a lognormal process

$$
S_{t}=S_{0} e^{\left(r-q-\frac{1}{2} \sigma^{2}\right) t+\sigma w_{t}},
$$

where $S_{0}$ is the initial stock price, $r$ is the risk-free rate, $q$ is the continuous dividend yield, $\sigma$ is the volatility of the underlying stock, $w_{t}$ is a standard Wiener process (Brownian motion). The three parameters $r, q$ and $\sigma$ are assumed to be constant.

The owner of an American put has a right to claim the difference between the strike price, $K$, and the stock price, $S_{t}$, at any time, $t$, before maturity, $T$. Therefore, the American put price has a lower bound of $P_{t} \geq \max \left(K-S_{t}, 0\right)$. Pricing an American put option involves two steps. The first step is to determine the critical stock price, $B_{t}^{p}$, which is a function of time, $t$. If $S_{t} \leq B_{t}^{p}$, one should exercise the American put. Otherwise, one should hold for a possible later exercise. The second step is to determine the price of the American put when exercising the put is not optimal. A standard argument shows that the price of an American put option, $P(S, t)$, satisfies the following Black-Scholes partial differential equation, boundary conditions and final conditions:

$$
\begin{aligned}
& \frac{\partial P}{\partial t}+\frac{1}{2} \sigma^{2} S^{2} \frac{\partial^{2} P}{\partial S^{2}}+(r-q) S \frac{\partial P}{\partial S}-r P=0, \quad \text { for } t<T, \quad S>B_{t}^{p}, \\
& P(S, t)=K-S, \quad \frac{\partial P}{\partial S}(S, t)=-1, \quad \text { for } t<T, \quad 0<S \leq B_{t}^{p}, \\
& P(S, T)=\max (K-S, 0), \\
& B_{T}^{p}=\left\{\begin{array}{ll}
K & \text { if } r \geq q \\
r & \text { if } r<q \\
q &
\end{array} .\right.
\end{aligned}
$$

The first two equations, (2) and (3), can be combined to give

$$
\frac{\partial P}{\partial t}+\frac{1}{2} \sigma^{2} S^{2} \frac{\partial^{2} P}{\partial S^{2}}+(r-q) S \frac{\partial P}{\partial S}-r P=\left\{\begin{array}{ll}
0 & \text { if } S>B_{t}^{p} \\
q S-r K & \text { if } S \leq B_{t}^{p}
\end{array}, \quad \text { for } t<T .\right.
$$

The backward inhomogeneous linear diffusion equation with the final condition (4) has the following solution:

$$
P(S, t)=p_{E}(S, t)+\int_{t}^{T}\left[r K e^{-r s} N\left(-d_{2}\left(S, B_{s}^{p}, s\right)\right)-q S e^{-q s} N\left(-d_{1}\left(S, B_{s}^{p}, s\right)\right)\right] d s,
$$


where $p_{E}$ is the price of the corresponding European put option given by the Black-Scholes (1973) formula,

$$
p_{E}(S, t)=K e^{-r(T-t)} N\left(-d_{2}(S, K, T-t)\right)-S e^{-q(T-t)} N\left(-d_{1}(S, K, T-t)\right),
$$

where $N(x)$, defined by

$$
N(x)=\frac{1}{\sqrt{2 \pi}} \int_{-\infty}^{x} e^{-\frac{1}{2} y^{2}} d y
$$

is the cumulative normal distribution function and

$$
d_{1}(x, y, t)=\frac{\ln (x / y)+\left(r-q+\frac{1}{2} \sigma^{2}\right) t}{\sigma \sqrt{t}}, \quad d_{2}(x, y, t)=d_{1}(x, y, t)-\sigma \sqrt{t}
$$

Equation (7) expresses the value of an American put as the sum of the value of a European put and the early-exercise premium. The early-exercise premium can be viewed as the value of a contingent claim that allows dividends paid by the stock, $q S d t$, to be exchanged for interest earned on the exercise price, $r K d t$, whenever the stock price is below the optimal exercise boundary. The expression for $B_{t}^{p}$ is crucial when we evaluate the integration in equation (7). We are unable to price an American put without a formula for the critical stock price. Applying equation (7) at the boundary, $S=B_{t}^{P}$, gives us a single integral equation

$$
\begin{aligned}
K-B_{t}^{p}= & K e^{-r(T-t)} N\left(-d_{2}\left(B_{t}^{p}, K, T-t\right)\right)-B_{t}^{p} e^{-q(T-t)} N\left(-d_{1}\left(B_{t}^{p}, K, T-t\right)\right) \\
& +\int_{t}^{T}\left[r K e^{-r s} N\left(-d_{2}\left(B_{t}^{p}, B_{s}^{p}, s\right)\right)-q B_{t}^{p} e^{-q s} N\left(-d_{1}\left(B_{t}^{p}, B_{s}^{p}, s\right)\right)\right] d s .
\end{aligned}
$$

One may notice that $B_{t}^{p}=0$ if $r=0$. One should never exercise an American put if the interest rate is zero.

The owner of an American call has a right to claim the difference between the stock price, $S_{t}$, and the strike price, $K$, at any time, $t$, before maturity, $T$. Therefore the American call price has a lower bound, $C_{t} \geq \max \left(S_{t}-K, 0\right)$. Pricing an American call option also involves two steps. The first step is to determine the critical stock price, $B_{t}^{c}$. If $S_{t} \geq B_{t}^{c}$, one should exercise the American call. Otherwise, one should hold for a possible later exercise. The second step is to determine the price of the American call when exercising 
the call is not optimal. The price of an American call option, $C(S, t)$, satisfies the following Black-Scholes partial differential equation, boundary conditions and final conditions:

$$
\begin{aligned}
& \frac{\partial C}{\partial t}+\frac{1}{2} \sigma^{2} S^{2} \frac{\partial^{2} C}{\partial S^{2}}+(r-q) S \frac{\partial C}{\partial S}-r C=0, \quad \text { for } t<T, \quad 0<S<B_{t}^{c}, \\
& C(S, t)=S-K, \quad \frac{\partial C}{\partial S}(S, t)=1, \quad \text { for } t<T, \quad S \geq B_{t}^{c} \\
& C(0, t)=0, \\
& C(S, T)=\max (S-K, 0), \\
& B_{T}^{c}= \begin{cases}K & \text { if } r \leq q \\
\frac{r}{q} K & \text { if } r>q\end{cases}
\end{aligned}
$$

The solution to the problem can be written in an integral form:

$$
C(S, t)=c_{E}(S, t)+\int_{t}^{T}\left[q S e^{-q s} N\left(d_{1}\left(S, B_{s}^{c}, s\right)\right)-r K e^{-r s} N\left(d_{2}\left(S, B_{s}^{c}, s\right)\right)\right] d s,
$$

where $c_{E}$ is the price of the corresponding European call option given by the Black-Scholes (1973) formula. The critical stock price, $B_{t}^{c}$, for the American call satisfies the following integral equation:

$$
\begin{aligned}
B_{t}^{c}-K= & B_{t}^{c} e^{-q(T-t)} N\left(d_{1}\left(B_{t}^{c}, K, T-t\right)\right)-K e^{-r(T-t)} N\left(d_{2}\left(B_{t}^{c}, K, T-t\right)\right) \\
& +\int_{t}^{T}\left[q B_{t}^{c} e^{-q s} N\left(d_{1}\left(B_{t}^{c}, B_{s}^{c}, s\right)\right)-r K e^{-r s} N\left(d_{2}\left(B_{t}^{c}, B_{s}^{c}, s\right)\right)\right] d s
\end{aligned}
$$

This equation has the property: if $q \rightarrow 0, B_{t}^{c} \rightarrow+\infty$, which leads to the well-known result that one should never exercise an American call if the underlying stock does not pay any dividend.

The analytical formula, (7) or (17), of the American option price was first introduced to the mathematics literature by Kolodner (1956) to study change of phase, and to the economics literature by McKean (1965). It has been restudied by Kim (1990), Jacka (1991), and Carr, Jarrow and Myneni (1992) to gain financial insights into the context of American option pricing. The American option pricing problem boils down to a mathematical problem of solving the integral equation, (11) or (18), for the critical stock price, $B_{t}^{p}$ or $B_{t}^{c}$. Huang, Subrahmanyam and Yu (1996) solve the optimal exercise boundary with a recursive numerical integration approach. Ju (1998) solves the problem by approximating 
the early-exercise boundary as a multi-piece exponential function. We solve the problem with a perturbation method. Our target is to obtain an analytical formula for the critical stock price.

\section{The Main Theorems}

Our main results are summarized in the following two theorems.

Theorem 1. The price of an American put option is given by the analytical formula (7) where the critical stock price, $B_{t}^{p}(r, q, \sigma, K, T)$, is given as follows:

If $r>q \geq 0$,

$$
\begin{aligned}
& B_{t}^{p}=K e^{-\sqrt{2 \sigma^{2}(T-t) u(\xi)}} \\
& u(\xi)=-\xi-\frac{1}{2 \xi}+\frac{1}{8 \xi^{2}}+\frac{11}{24 \xi^{3}}+O\left(\frac{1}{\xi^{4}}\right), \\
& \xi=\ln \sqrt{8 \pi(r-q)^{2}(T-t) / \sigma^{2}} .
\end{aligned}
$$

If $r=q$,

$$
\begin{aligned}
& B_{t}^{p}=K e^{-\sqrt{2 \sigma^{2}(T-t) v(\eta)}}, \\
& v(\eta)=-\eta-\frac{1}{2} \ln (-\eta)-\frac{1}{4 \eta} \ln (-\eta)-\frac{1-\frac{5}{4 \sqrt{2 \pi}}}{\eta}+o\left(\frac{1}{\eta}\right), \\
& \eta=\ln [4 \sqrt{\pi} r(T-t)] .
\end{aligned}
$$

If $r<q$,

$$
\begin{aligned}
& B_{t}^{p}=\frac{r}{q} K e^{-2 \sqrt{\tau^{*}} w\left(\sqrt{\tau^{*}}\right)}, \quad \tau^{*}=\frac{1}{2} \sigma^{2}(T-t), \quad r^{*}=\frac{r}{\frac{1}{2} \sigma^{2}}, \quad q^{*}=\frac{q}{\frac{1}{2} \sigma^{2}}, \\
& w\left(\sqrt{\tau^{*}}\right)=\beta_{0}+\beta_{1} \sqrt{\tau^{*}}+\beta_{2} \tau^{*}+\beta_{3} \tau^{* 3 / 2}+O\left(\tau^{* 2}\right), \\
& \beta_{0}=0.451723, \quad \beta_{1}=0.144914\left(r^{*}-q^{*}\right), \\
& \beta_{2}=-0.009801-0.041764\left(r^{*}+q^{*}\right)+0.014829\left(r^{*}-q^{*}\right)^{2}, \\
& \beta_{3}=-0.000618-0.002087\left(r^{*}-q^{*}\right)-0.015670\left(r^{* 2}-q^{* 2}\right)-0.001052\left(r^{*}-q^{*}\right)^{3} .
\end{aligned}
$$

The proof of Theorem 1 is given in Appendix A.

Remark 1. The present formulas of $u(\xi), v(\eta)$ and $w\left(\sqrt{\tau^{*}}\right)$ are derived with a perturbation method under the assumption of small $\sigma^{2} T$. It covers almost all the existing approximate 
formulas in the same family as a special case. For example, Chen and Chadam (2007) provide a formula for $u(\xi)$ with $q=0$. Evans, Kuske and Keller (2002) provide the first terms of the present formulas for $u(\xi), v(\eta)$ and $w\left(\sqrt{\tau^{*}}\right)$.

Theorem 2. The price of an American call option is given by the analytical formula (17) where the critical stock price, $B_{t}^{c}(r, q, \sigma, K, T)$, is given by the following duality relation:

$$
B_{t}^{c}(r, q, \sigma, K, T)=\frac{K^{2}}{B_{t}^{p}(q, r, \sigma, K, T)}
$$

or explicitly as follows:

If $r>q \geq 0$,

$$
\begin{aligned}
& B_{t}^{c}=\frac{r}{q} K e^{2 \sqrt{\tau^{*}} w\left(\sqrt{\tau^{*}}\right)}, \quad \tau^{*}=\frac{1}{2} \sigma^{2}(T-t), \quad r^{*}=\frac{r}{\frac{1}{2} \sigma^{2}}, \quad q^{*}=\frac{q}{\frac{1}{2} \sigma^{2}}, \\
& w\left(\sqrt{\tau^{*}}\right)=\beta_{0}+\beta_{1} \sqrt{\tau^{*}}+\beta_{2} \tau^{*}+\beta_{3} \tau^{* 3 / 2}+O\left(\tau^{* 2}\right), \\
& \beta_{0}=0.451723, \quad \beta_{1}=0.144914\left(q^{*}-r^{*}\right), \\
& \beta_{2}=-0.009801-0.041764\left(q^{*}+r^{*}\right)+0.014829\left(q^{*}-r^{*}\right)^{2}, \\
& \beta_{3}=-0.000618-0.002087\left(q^{*}-r^{*}\right)-0.015670\left(q^{* 2}-r^{* 2}\right)-0.001052\left(q^{*}-r^{*}\right)^{3} .
\end{aligned}
$$

If $r=q$,

$$
\begin{aligned}
& B_{t}^{c}=K e^{\sqrt{2 \sigma^{2}(T-t) v(\eta)}}, \\
& v(\eta)=-\eta-\frac{1}{2} \ln (-\eta)-\frac{1}{4 \eta} \ln (-\eta)-\frac{1-\frac{5}{4 \sqrt{2 \pi}}}{\eta}+o\left(\frac{1}{\eta}\right), \\
& \eta=\ln [4 \sqrt{\pi} r(T-t)] .
\end{aligned}
$$

If $r<q$,

$$
\begin{aligned}
& B_{t}^{c}=K e^{\sqrt{2 \sigma^{2}(T-t) u(\xi)}}, \\
& u(\xi)=-\xi-\frac{1}{2 \xi}+\frac{1}{8 \xi^{2}}+\frac{11}{24 \xi^{3}}+O\left(\frac{1}{\xi^{4}}\right), \\
& \xi=\ln \sqrt{8 \pi(r-q)^{2}(T-t) / \sigma^{2}} .
\end{aligned}
$$

The proof of Theorem 2 is given in Appendix B. 
Remark 1. It seems to us that the duality relation (22) between the critical stock price of an American call and that of an American put with the same strike is not well known. Evans, Kuske and Keller (2002) study the problem of the American call with asymptotic analysis and provide the first terms of our formulas $u(\xi), v(\eta)$ and $w\left(\sqrt{\tau^{*}}\right)$.

Remark 2. In principle, one is able to push the series of $u(\xi), v(\eta)$ and $w\left(\sqrt{\tau^{*}}\right)$ to any higher order, but it involves much more algebra. We choose to stop at the level of the fourth-order term and go on to test the accuracy of the formulas. The formulas are derived theoretically with an assumption of small $\sigma^{2} T$. How small is it practically? This important question has to be answered before academics and market participants can use the formula. A numerical test on the accuracy of the formulas must be performed in order to answer the question.

\section{The Computation of Highly Accurate Numerical Values of the Critical Stock Price and the Amer- ican Option Price}

In order to test the accuracy of the present formulas, we need highly accurate numerical values as a benchmark. There are many different ways to compute the critical stock price and the American option price numerically. The most popular ones include the binomialtree method, the PDE method and the integral-equation approach. We adopt the integralequation approach in this paper because it computes the critical stock price directly.

Highly accurate numerical values of the critical stock price of an American put can be computed by solving the integral equation (11) numerically. For a given set of parameters, $(r, q, \sigma, K, T)$, in order to solve the equation for $B^{p}$ at a particular time, $t$, we need $B^{p}$ for the time interval between $t$ and $T$. Therefore, we need to solve $B^{p}$ backward from $T$, where $B_{T}^{p}$ is known to be $K$ if $r \geq q$ or $(r / q) K$ if $r<q$. For example, at time $T-\Delta t, B_{T-\Delta t}^{p}$ solves the following equation:

$K-B_{T-\Delta t}^{p}=p_{E}\left(B_{T-\Delta t}^{p}, T-\Delta t\right)+\frac{1}{2}\left[f\left(B_{T-\Delta t}^{p}, B_{T-\Delta t}^{p}, T-\Delta t\right)+f\left(B_{T-\Delta t}^{p}, B_{T}^{p}, T\right)\right] \Delta t$, 
where

$$
f\left(B_{t}^{p}, B_{s}^{p}, s\right)=r K e^{-r s} N\left(-d_{2}\left(B_{t}^{p}, B_{s}^{p}, s\right)\right)-q B_{t}^{p} e^{-q s} N\left(-d_{1}\left(B_{t}^{p}, B_{s}^{p}, s\right)\right)
$$

and a trapezoidal rule is used in the numerical integration. The equation can be solved numerically with a standard root-finding algorithm. Once $B_{T-\Delta t}^{p}$ is known, $B_{T-2 \Delta t}^{p}$ can be found by solving the following equation

$$
\begin{array}{r}
K-B_{T-2 \Delta t}^{p}=p_{E}\left(B_{T-2 \Delta t}^{p}, T-2 \Delta t\right)+\frac{1}{2}\left[f\left(B_{T-2 \Delta t}^{p}, B_{T-2 \Delta t}^{p}, T-2 \Delta t\right)+\right. \\
\left.2 f\left(B_{T-2 \Delta t}^{p}, B_{T-\Delta t}^{p}, T-\Delta t\right)+f\left(B_{T-2 \Delta t}^{p}, B_{T}^{p}, T\right)\right] \Delta t .
\end{array}
$$

Once $B_{T-i \Delta t}^{p}, i=1,2, \cdots, n-1$, are known, $B_{T-n \Delta t}^{p}$ can be found by solving

$$
\begin{array}{r}
K-B_{T-n \Delta t}^{p}=p_{E}\left(B_{T-n \Delta t}^{p}, T-n \Delta t\right)+\frac{1}{2}\left[f\left(B_{T-n \Delta t}^{p}, B_{T-n \Delta t}^{p}, T-n \Delta t\right)+\right. \\
\left.2 \sum_{i=1}^{n-1} f\left(B_{T-n \Delta t}^{p}, B_{T-(n-i) \Delta t}^{p}, T-(n-i) \Delta t\right)+f\left(B_{T-n \Delta t}^{p}, B_{T}^{p}, T\right)\right] \Delta t .
\end{array}
$$

We have solved $B_{T-i \Delta t}^{p}, i=1,2, \cdots, n$, successively. With all the information on the boundary, we can price the American put at time $t=T-n \Delta t$ by computing the integration formula (7) numerically, i.e.,

$$
\begin{array}{r}
P(S, T-n \Delta t)=p_{E}(S, T-n \Delta t)+\frac{1}{2}\left[f\left(S, B_{T-n \Delta t}^{p}, T-n \Delta t\right)+\right. \\
\left.\quad 2 \sum_{i=1}^{n-1} f\left(S, B_{T-(n-i) \Delta t}^{p}, T-(n-i) \Delta t\right)+f\left(S, B_{T}^{p}, T\right)\right] \Delta t .
\end{array}
$$

The numerical results are presented in Tables 1 and 2. Table 1 is the critical stock price of an American put option as a function of the time to maturity from a week up to 17 weeks. As the grid size reduces from a week to half of a week, to a quarter of a week, $1 / 8$ of a week, $\cdots, 1 / 64$ of a week, the computed numerical values converge to the true value with an error of 0.01 cent for an option with up to three month maturity and a strike price of 100 dollars. Table 2 shows the American put prices for a range of moneyness from $-10 \%$ to $10 \%$ and volatility from 0.2 to 0.5 . The computed prices of a put option with one-month maturity ${ }^{5}$ are also convergent with an error of 0.01 cent as the time grid size decreases from $1 / 20$ of a month to $1 / 320$ of a month.

\footnotetext{
${ }^{5}$ Most of the time, our test is restricted to a one-month maturity in this paper, partly because most liquidly traded American-style OEX options have a maturity of about a month.
} 
With the highly accurate numerical values computed, we are now able to test the accuracy of the present formulas with certain truncated terms.

\section{Numerical Test on the Accuracy of the Present For- mulas}

Since the American call and put have a duality relationship, we only need to test the formulas for the American put. The conclusion on the accuracy of the American put price automatically applies to that of the American call price.

We have done many numerical tests for different values of parameters $(r, q)$. We find out that the American option price errors are quite stable in each region of $r>q \geq 0$, $r=q$ and $r<q$. We now present one typical case in each region to illustrate the errors.

The results for $r>q \geq 0$ are presented in Tables 3 and 4 . Table 3 is the critical stock price as a function of the time to maturity from one week up to four months. The first column shows the highly accurate numerical values computed from the integral equation. The other four columns are computed by using the present analytical formulas with one, two, three and four truncated terms. The convergency of the series becomes an issue. Intuitively the series for $u(\xi)$ converges for large $|\xi|$. Based on the numerical values in Table 3, the series converges for small $T-t$, e.g., $T-t=1 / 12$, which corresponds to $\xi=-1.42213$. It does not converge for large $T-t$, e.g., $T-t=1 / 3$, which corresponds to $\xi=-0.72898$. The condition of convergency is under investigation. Table 4 is the price of the American options with a one-month maturity and a strike price of 100 dollars for a range of moneyness from $-10 \%$ to $10 \%$ and volatility from 0.2 to 0.5 . Compared with the highly accurate numerical values computed with the numerical-integration method described in $\S 4$, the present formulas give very accurate prices. For example, the formula with the first two terms gives a price with an error of 0.07 cent. This corresponds to OEX options with an error of 0.3 cent, since OEX options have a strike price of about 400 dollars (the S\&P 100 index level on March 12, 2003 was $\$ 408.92)$. The accuracy is good enough for practical application. The price given by the present formula can be treated as the true model price 
if it is used to price OEX options.

The results for $r=q$ are presented in Tables 5 and 6 . The series of the critical stock price in Table 5 converges for small $T-t$, but not for large $T-t$. But the convergency is slightly better than that for the case of $r>q$ in Table 3, because of the fact that the singularity near expiration in this case is weaker than that in the last case. The accuracy of the American option price in Table 6 is also better than that for the case $r>q$ in Table 4. For example, the formula with the first four terms gives a price with an error of 0.03 cent. This corresponds to OEX options with an error of 0.1 cent.

The results for $r<q$ are presented in Tables 7 and 8 . The critical stock price in Table 7 computed with the present formulas converges to a value that is different from the highly accurate numerical value. We are still investigating why there is such a difference. The American option prices in Table 8 are almost identical to the highly accurate numerical values. The error, even with only the first term, is only 0.01 cent, which corresponds to 0.04 cent for OEX options. The accuracy of the formula in this case is the best of the three cases due to its weak singularity near expiration. Our results suggest that the formula in this case has the potential to price American options with longer maturities. A more comprehensive test will be reported in a subsequent study on developing an analytical formula for the critical stock price of a long-term American option.

The solutions obtained for the regions $r>q \geq 0, r=q$ and $r<q$ are totally different. To further test the continuity of three formulas (19), (20) and (21) in Theorem 1 near the neighborhood of $|r-q| \ll 1$, we present the results of $(r, q)=(0.05,0.0499),(r, q)=$ $(0.05,0.05)$ and $(r, q)=(0.05,0.0501)$ in Table 9. The computed American option prices are indeed very close with three different critical-stock-price formulas. The relative difference is smaller than $0.3 \%$. The accuracy is good enough for the application of pricing OEX options.

Our study shows that further research is required to enhance the convergency and accuracy of the series for the critical stock price. The key is to find some other ways to expand the two functions $u(\xi)$ and $v(\eta)$, so that the series converge for large value of $T-t$. 
This is a problem for further research.

\section{Conclusion}

Pricing American options has been an outstanding issue in finance for thirty years, since the no-arbitrage option pricing model was established by Black and Scholes (1973) and Merton (1973). The difficulty comes from the early-exercise feature in the contract. Analytically describing the critical stock price is challenging.

This paper solves the problem near expiration by using a perturbation method with an assumption of small $\sigma^{2} T$. We have obtained an analytical formula in a series form for the critical stock price of American option. We also present a duality relationship between the critical stock price of an American call and that of an American put with the same strike price. With the present analytical formula, one is able to price and hedge American options by using the analytical integration formula. We have also performed comprehensive numerical tests on the accuracy of the early-exercise boundary and the option price computed by using the present formulas with some truncated terms. The numerical tests show that our formula with up to four terms, one or two terms in some cases, is sufficient in pricing short-term American options with maturities of one or two months. The error is under half a cent if the formula is used to price liquidly traded OEX options with a maturity of about one month, a strike price of about 400 dollars, moneyness under $10 \%$ and underlying volatility from 0.2 to 0.5 . The present formula is now ready for traders to use in pricing OEX options and for the CBOE to use in computing VXO, since the volatility index is defined as the implied volatility of one-month at-the-money OEX options.

The perturbation method presented in this paper can be used to study the price of other derivatives ${ }^{6}$ and convertible bonds with some embedded American options. The extension of the present method to price a long-term American option, such as long-term equity anticipation securities (LEAPS) with maturities up to three years, is left for further

\footnotetext{
${ }^{6}$ For example, Zhang (2003) studies the price of continuously sampled Asian options by using the perturbation method.
} 
research.

\section{Appendix}

\section{A Proof of Theorem 1}

Applying a standard transformation $S \leftarrow x, t \leftarrow t^{*}, P(S, t) \leftarrow p\left(x, t^{*}\right)$ and $B_{t}^{p} \leftarrow s\left(t^{*}\right)$ as

$$
S=K e^{x}, \quad t=T-\frac{2}{\sigma^{2}} t^{*}, \quad P(S, t)=K p\left(x, t^{*}\right), \quad B_{t}^{p}=K e^{s\left(t^{*}\right)},
$$

to equations $(2,3,4,5)$ yields

$$
\left\{\begin{array}{l}
p_{t^{*}}-p_{x x}-\left(r^{*}-q^{*}-1\right) p_{x}+r^{*} p=0, \quad \text { for } t^{*}>0, \quad x>s\left(t^{*}\right), \\
p\left(x, t^{*}\right)=1-e^{x}, \quad p_{x}\left(x, t^{*}\right)=-e^{x}, \quad \text { for } t^{*}>0, \quad-\infty<x \leq s\left(t^{*}\right), \\
p(x, 0)=\max \left(1-e^{x}, 0\right), \quad \text { for }-\infty<x<\infty, \\
s(0)= \begin{cases}0 \quad \text { if } r^{*} \geq q^{*} \\
\ln \frac{r^{*}}{q^{*}} \text { if } r^{*}<q^{*}\end{cases}
\end{array}\right.
$$

where

$$
r^{*}=\frac{2 r}{\sigma^{2}}, \quad q^{*}=\frac{2 q}{\sigma^{2}} .
$$

For simplicity of notation, from now on, we drop the star in variables $t^{*}, r^{*}$ and $q^{*}$ while keeping in mind that they denote the dimensionless time to maturity, interest rate and dividend yield. We denote by $\mathcal{L}$ the operator

$$
\mathcal{L}[p]=p_{x x}+(r-q-1) p_{x}-r p,
$$

and by $\Gamma(x, t)$ the fundamental solution to the operator $\partial_{t}-\mathcal{L}$, more precisely

$$
\Gamma(x, t)=\frac{1}{2 \sqrt{\pi t}} \exp \left\{-\frac{[x+(r-q-1) t]^{2}}{4 t}-r t\right\} .
$$

Applying Green's identity to equation (23) gives us a formula for the American put option price, $p(x, t)$, in terms of the free boundary $s(t)$,

$p(x, t)=\int_{-\infty}^{0}\left(1-e^{y}\right) \Gamma(x-y, t) d y+\int_{0}^{t} \int_{-\infty}^{s(t-\tau)}\left(r-q e^{y}\right) \Gamma(x-y, \tau) d y d \tau, \quad x \in R, t>0,(26)$ 
where the first term gives the Black-Scholes formula for the European put and the second term is the early-exercise premium. The double integration in the second term can not be carried out without knowledge of the free boundary. The formula is equivalent to equation (7) presented in the paper.

In order to solve the free boundary analytically, we need an equation for the boundary only. We now construct a few such equations. Since $\Gamma(\cdot, 0)$ is the Delta function centered at the origin, using

$$
\begin{aligned}
\Gamma_{\tau}(x-y, \tau) & =\Gamma_{x x}(x-y, \tau)+(r-q-1) \Gamma_{x}(x-y, \tau)-r \Gamma(x-y, \tau) \\
& =\Gamma_{y y}(x-y, \tau)-(r-q-1) \Gamma_{y}(x-y, \tau)-r \Gamma(x-y, \tau)
\end{aligned}
$$

and integrating by parts, we have the following equality:

$$
\begin{array}{r}
\int_{-\infty}^{0}\left(1-e^{y}\right) \Gamma(x-y, t) d y=\max \left(1-e^{x}, 0\right)+\int_{0}^{t} \int_{-\infty}^{0}\left(1-e^{y}\right) \Gamma_{\tau}(x-y, \tau) d y d \tau \\
=\max \left(1-e^{x}, 0\right)+\int_{0}^{t}\left[\Gamma(x, \tau)-\int_{-\infty}^{0} \Gamma(x-y, \tau)\left(r-q e^{y}\right) d y\right] d \tau .
\end{array}
$$

Substituting the identity into (26) gives us the following option pricing formula for $x \in R$ and $t>0$ :

$$
p(x, t)=\max \left(1-e^{x}, 0\right)+\int_{0}^{t}\left[\Gamma(x, \tau)-\int_{s(t-\tau)}^{0}\left(r-q e^{y}\right) \Gamma(x-y, \tau) d y\right] d \tau,
$$

where the first term is the intrinsic value of the American put and the second term is its time value. If the second term is zero, the American put should be exercised immediately. Differentiating (28) with respect to $t$ yields

$$
\begin{aligned}
p_{t}(x, t)= & \Gamma(x, t)-\int_{s(0)}^{0}\left(r-q e^{y}\right) \Gamma(x-y, t) d y \\
& +\int_{0}^{t}\left(r-q e^{s(t-\tau)}\right) \Gamma(x-s(t-\tau), \tau) \dot{s}(t-\tau) d \tau .
\end{aligned}
$$

Differentiating (28) with respect to $x$ and $t$ yields

$$
\begin{aligned}
p_{x t}(x, t)= & \Gamma_{x}(x, t)-\int_{s(0)}^{0}\left(r-q e^{y}\right) \Gamma_{x}(x-y, t) d y \\
& +\int_{0}^{t}\left(r-q e^{s(t-\tau)}\right) \Gamma_{x}(x-s(t-\tau), \tau) \dot{s}(t-\tau) d \tau .
\end{aligned}
$$


At the free boundary, $p(x, t)$ and $p_{x}(x, t)$ are continuous. In fact,

$$
\begin{aligned}
& \left.p(x, t)\right|_{x=s^{+}(t)}=\left.p(x, t)\right|_{x=s^{-}(t)}=\left.\left(1-e^{x}\right)\right|_{x=s^{-}(t)}=1-e^{s(t)}, \\
& \left.p_{x}(x, t)\right|_{x=s^{+}(t)}=\left.p_{x}(x, t)\right|_{x=s^{-}(t)}=-\left.e^{x}\right|_{x=s^{-}(t)}=-e^{s(t)} .
\end{aligned}
$$

Taking total differentiation of (31) and (32) with respect to $t$ at the boundary yields

$$
\begin{aligned}
& \left.\dot{s}(t) p_{x}(x, t)\right|_{x=s^{+}(t)}+\left.p_{t}(x, t)\right|_{x=s^{+}(t)}=-\dot{s}(t) e^{s(t)} \\
& \left.\dot{s}(t) p_{x x}(x, t)\right|_{x=s^{+}(t)}+\left.p_{x t}(x, t)\right|_{x=s^{+}(t)}=-\dot{s}(t) e^{s(t)}
\end{aligned}
$$

Substituting equation (32) into (33) gives

$$
\left.p_{t}(x, t)\right|_{x=s^{+}(t)}=0 .
$$

Taking the limit $x \rightarrow s^{+}(t)$ of the first equation in (23) gives

$$
\begin{aligned}
\left.p_{x x}(x, t)\right|_{x=s^{+}(t)} & =\left.\left[p_{t}(x, t)-(r-q-1) p_{x}(x, t)+r p(x, t)\right]\right|_{x=s^{+}(t)} \\
& =0+(r-q-1) e^{s(t)}+r\left(1-e^{s(t)}\right)=r-(q+1) e^{s(t)} .
\end{aligned}
$$

Substituting equation (36) into (34) gives

$$
\left.p_{x t}(x, t)\right|_{x=s^{+}(t)}=-\left(r-q e^{s(t)}\right) \dot{s}(t) .
$$

Applying the two equations $(29,30)$ at the free boundary, $x=s^{+}(t)$, and using the two conditions in equations (35) and (37), we have

$$
\begin{aligned}
\Gamma(s(t), t)= & \int_{s(0)}^{0}\left(r-q e^{y}\right) \Gamma(s(t)-y, t) d y \\
& -\int_{0}^{t}\left(r-q e^{s(t-\tau)}\right) \Gamma(s(t)-s(t-\tau), \tau) \dot{s}(t-\tau) d \tau \\
\left(r-q e^{s(t)}\right) \dot{s}(t)= & -2 \Gamma_{x}(s(t), t)+2 \int_{s(0)}^{0}\left(r-q e^{y}\right) \Gamma_{x}(s(t)-y, t) d y \\
& -2 \int_{0}^{t}\left(r-q e^{s(t-\tau)}\right) \Gamma_{x}(s(t)-s(t-\tau), \tau) \dot{s}(t-\tau) d \tau .
\end{aligned}
$$

In taking the limit for $p_{x t}$, we need the following fact (see, e.g., Cannon 1984, Lemma 14.2.3.-14.2.5., pp. 218-223): for any continuous function $f$,

$$
\lim _{x \rightarrow s^{+}(t)} \int_{0}^{t} f(t-\tau) \Gamma_{x}(x-s(t-\tau), \tau) d \tau=-\frac{f(t)}{2}+\int_{0}^{t} f(t-\tau) \Gamma_{x}(s(t)-s(t-\tau), \tau) d \tau .
$$


Since

$$
\Gamma_{x}(x, t)=-\frac{x+(r-q-1) t}{2 t} \Gamma(x, t),
$$

adding equations (39) and (38) multiplied by $[s(t)-s(0)+2(r-q-1) t] /(2 t)$ gives

$$
\begin{aligned}
\left(r-q e^{s(t)}\right) \dot{s}(t)= & \frac{s(t)+s(0)}{2 t} \Gamma(s(t), t) \\
& -\int_{s(0)}^{0}\left(r-q e^{y}\right)\left[\frac{s(t)-y}{t}-\frac{s(t)-s(0)}{2 t}\right] \Gamma(s(t)-y, t) d y \\
& +\int_{0}^{t}\left(r-q e^{s(t-\tau)}\right)\left[\frac{s(t)-s(t-\tau)}{\tau}-\frac{s(t)-s(0)}{2 t}\right] \\
& \Gamma(s(t)-s(t-\tau), \tau) \dot{s}(t-\tau) d \tau .
\end{aligned}
$$

This is the integro-differential equation that we use to solve for the free boundary, $s(t)$. The equation for the special case without a dividend, i.e., $q=0$, was derived and used by Chen and Chadam (2007).

Now, we study the asymptotic solution of the free boundary near expiration for three cases: $r>q \geq 0, r=q$ and $r<q$.

\section{A.1 Case 1: $r>q \geq 0$}

For this case, $s(0)=0$, equation (40) becomes

$$
\begin{array}{r}
\left(r-q e^{s(t)}\right) \dot{s}(t)=\frac{s(t)}{2 t} \Gamma(s(t), t)+\int_{0}^{t}\left(r-q e^{s(t-\tau)}\right)\left[\frac{s(t)-s(t-\tau)}{\tau}-\frac{s(t)}{2 t}\right] \\
\Gamma(s(t)-s(t-\tau), \tau) \dot{s}(t-\tau) d \tau,
\end{array}
$$

or

$$
\begin{array}{r}
s(t)\left[\dot{s}(t)-\frac{s(t)}{2 t\left(r-q e^{s(t)}\right)} \Gamma(s(t), t)\right]=s(t) \int_{0}^{t} \frac{r-q e^{s(t-\tau)}}{r-q e^{s(t)}}\left[\frac{s(t)-s(t-\tau)}{\tau}-\frac{s(t)}{2 t}\right] \\
\Gamma(s(t)-s(t-\tau), \tau) \dot{s}(t-\tau) d \tau .
\end{array}
$$

Setting

$$
s(t)=-\sqrt{4 u(\xi) t}, \quad \xi=\ln \sqrt{4 \pi(r-q)^{2} t},
$$

by assuming $\tau=\frac{4 z t}{(1+z)^{2}}$, we transform equation (42) into the new variables $(u, \xi)$,

$$
u^{\prime}+2 u\left(1-e^{-u-\xi+a}\right)=-\int_{0}^{1}\left(\frac{1+z^{2}}{z} \sqrt{u}-\frac{1-z^{2}}{z} \sqrt{\hat{u}}\right)\left(1+\frac{\hat{u}^{\prime}}{2 \hat{u}}\right) \frac{\sqrt{-\xi \hat{u}}}{\sqrt{\pi z}} e^{\xi z-b} d z
$$


or

$u=-\xi-\ln \left\{1+\frac{u^{\prime}}{2 u}+\frac{1}{2 u} \int_{0}^{1}\left(\frac{1+z^{2}}{z} \sqrt{u}-\frac{1-z^{2}}{z} \sqrt{\hat{u}}\right)\left(1+\frac{\hat{u}^{\prime}}{2 \hat{u}}\right) \frac{\sqrt{-\xi \hat{u}}}{\sqrt{\pi z}} e^{\xi z-b} d z\right\}+a$,

where $u=u(\xi), u^{\prime}=u^{\prime}(\xi), \hat{u}=u\left(\xi+\ln \frac{1-z}{1+z}\right), \hat{u}^{\prime}=u^{\prime}\left(\xi+\ln \frac{1-z}{1+z}\right)$, and

$$
\begin{aligned}
& a=\frac{(r-q-1) \sqrt{u} e^{\xi}}{2 \sqrt{\pi}(r-q)}-\frac{(r-q-1)^{2}+4 r}{16 \pi(r-q)^{2}} e^{2 \xi}-\ln \left(1+\frac{q}{r-q}\left(1-e^{-\frac{\sqrt{u} \xi^{\xi}}{\sqrt{\pi}(r-q)}}\right)\right), \\
& b=\ln \sqrt{\frac{-\xi}{u}}+(u+\xi) z+\ln (1+z)+\frac{1-z}{2}(u-\hat{u})+\frac{1-z^{2}}{4 z}(\sqrt{u}-\sqrt{\hat{u}})^{2} \\
& -\frac{(r-q-1) e^{\xi}}{2 \sqrt{\pi}(r-q)}\left(\sqrt{u}-\frac{1-z}{1+z} \sqrt{\hat{u}}\right)+\frac{\left[(r-q-1)^{2}+4 r\right] z e^{2 \xi}}{4(r-q)^{2} \pi(1+z)^{2}} \\
& -\ln \left(1+\frac{q}{r-q}\left(1-e^{-\frac{1-z}{1+z} \frac{\sqrt{u} \xi}{\sqrt{\pi}(r-q)}}\right)\right)+\ln \left(1+\frac{q}{r-q}\left(1-e^{-\frac{\sqrt{u} e}{\sqrt{\pi}(r-q)}}\right)\right) .
\end{aligned}
$$

The problem becomes similar to the case without dividends that has been studied by Chen and Chadam (2007). By starting with $u=-\xi$ and successively replacing $u$ on the righthand side of (45) by its previous expansion, we obtain the asymptotic expansion for $u(\xi)$ near $t=0$ :

$$
u(\xi)=-\xi+\sum_{i=1}^{\infty} \frac{\alpha_{i}}{\xi^{i}}=-\xi-\frac{1}{2 \xi}+\frac{1}{8 \xi^{2}}+\frac{11}{24 \xi^{3}}+O\left(\frac{1}{\xi^{4}}\right),
$$

The key here is that the right-hand side of (45) produces a unique $n+1^{\text {st }}$ order expansion, if an $n^{\text {th }}$ order expansion of $u$ is given, because of the denominator $2 u$.

\section{A.2 Case 2: $r=q$}

In this case, $r=q, s(0)=0$, equation (40) becomes

$$
\begin{array}{r}
r\left(1-e^{s(t)}\right) \dot{s}(t)=\frac{s(t)}{2 t} \Gamma(s(t), t)+\int_{0}^{t} r\left(1-e^{s(t-\tau)}\right)\left[\frac{s(t)-s(t-\tau)}{\tau}-\frac{s(t)}{2 t}\right] \\
\Gamma(s(t)-s(t-\tau), \tau) \dot{s}(t-\tau) d \tau
\end{array}
$$

or

$$
\begin{gathered}
\left(1-e^{s(t)}\right) \dot{s}(t)-\frac{s(t)}{2 t r} \Gamma(s(t), t)=\int_{0}^{t}\left(1-e^{s(t-\tau)}\right)\left[\frac{s(t)-s(t-\tau)}{\tau}-\frac{s(t)}{2 t}\right] \\
\Gamma(s(t)-s(t-\tau), \tau) \dot{s}(t-\tau) d \tau
\end{gathered}
$$

Setting

$$
s(t)=-\sqrt{4 v(\eta) t}, \quad \eta=\ln (4 t r \sqrt{\pi}),
$$


by assuming $\tau=\frac{2 z t}{1+z}$, we transform equation (48) into the new variables $(v, \eta)$,

$$
H+2 \sqrt{v} \exp [-v-\eta+c]=-\int_{0}^{1}\left(1+\frac{\hat{v}^{\prime}}{\hat{v}}\right) F d z
$$

or

$$
v=-\eta-\ln \left\{\frac{1}{2 \sqrt{v}}\left[-H-\int_{0}^{1}\left(1+\frac{\hat{v}^{\prime}}{\hat{v}}\right) F d z\right]\right\}+c
$$

where $v=v(\eta), v^{\prime}=v^{\prime}(\eta), \hat{v}=v\left(\eta+\ln \frac{1-z}{1+z}\right), \hat{v}^{\prime}=v^{\prime}\left(\eta+\ln \frac{1-z}{1+z}\right)$, and

$$
\begin{aligned}
& c=-\frac{\sqrt{v} e^{\frac{\eta}{2}}}{2 \sqrt{r \sqrt{\pi}}}-\frac{1+4 r}{16 r \sqrt{\pi}} e^{\eta} \\
& H=2 \sqrt{r \sqrt{\pi}} e^{-\frac{\eta}{2}}\left(e^{-\frac{\sqrt{v} e^{\frac{\eta}{2}}}{\sqrt{r \sqrt{\pi}}}}-1\right)\left(\sqrt{v}+\frac{v^{\prime}}{\sqrt{v}}\right), \\
& F=\sqrt{\frac{2 r}{\sqrt{\pi}}} \frac{e^{-\frac{\eta}{2}}}{(1+z) \sqrt{z(1-z)}}\left(1-e^{-\frac{\sqrt{\hat{v}}}{\sqrt{r \sqrt{\pi}}} e^{\frac{\eta}{2}} \sqrt{\frac{1-z}{1+z}}}\right) \sqrt{\hat{v}}\left[\sqrt{v}+\frac{1+z}{z}\left(\sqrt{\frac{1-z}{1+z} \sqrt{\hat{v}}}-\sqrt{v}\right)\right] \\
& \exp \left[-\frac{1+z}{2 z}\left(\sqrt{\frac{1-z}{1+z}} \sqrt{\hat{v}}-\sqrt{v}\right)^{2}+\frac{e^{\frac{\eta}{2}}}{2 \sqrt{r \sqrt{\pi}}}\left(\sqrt{\frac{1-z}{1+z}} \sqrt{\hat{v}}-\sqrt{v}\right)-\frac{(1+4 r) e^{\eta}}{8 r \sqrt{\pi}} \frac{z}{1+z}\right] .
\end{aligned}
$$

In the same way as in Case 1, we solve (51) by starting with $v=-\eta$ and successively replacing $v$ on the right-hand side by its previous expansion. We then obtain the asymptotic expansion for $v(\eta)$ near $t=0$ :

$$
v(\eta)=-\eta-\frac{1}{2} \ln (-\eta)-\frac{1}{4 \eta} \ln (-\eta)-\frac{1-\frac{5}{4 \sqrt{2 \pi}}}{\eta}+o\left(\frac{1}{\eta}\right) .
$$

\section{A.3 Case 3: $r<q$}

In this case, $s(0)=\ln r-\ln q<0$, equation (40) becomes

$$
\begin{aligned}
\left(1-e^{\tilde{s}(t)}\right) \dot{\tilde{s}}(t)= & \frac{\tilde{s}(t)+2 s(0)}{2 r t} \Gamma(\tilde{s}(t)+s(0), t) \\
& +\int_{0}^{t}\left(1-e^{\tilde{s}(t-\tau)}\right)\left[\frac{\tilde{s}(t)-\tilde{s}(t-\tau)}{\tau}-\frac{\tilde{s}(t)}{2 t}\right] \Gamma(\tilde{s}(t)-\tilde{s}(t-\tau), \tau) \dot{\tilde{s}}(t-\tau) d \tau \\
& -\int_{0}^{-s(0)}\left(1-e^{y}\right)\left[\frac{\tilde{s}(t)}{2 t}-\frac{y}{t}\right] \Gamma(\tilde{s}(t)-y, t) d y
\end{aligned}
$$

where $s(t)=s(0)+\tilde{s}(t)$. We adopt the following singular perturbation scheme for small $t$ :

$$
\tilde{s}(t)=-2 \sqrt{t} w(\sqrt{t}) .
$$


Since, for any fixed $\epsilon \in(0,1)$

$$
\frac{\tilde{s}(t)+2 s(0)}{2 r t} \Gamma(\tilde{s}(t)+s(0), t)=O\left(t^{-\frac{3}{2}}\right) e^{-\frac{\epsilon s^{2}(0)}{4 t}},
$$

we obtain from (53) the asymptotic expansion for $w(\sqrt{t})$ near $t=0$

$$
w(\sqrt{t})=\beta_{0}+\beta_{1} \sqrt{t}+\beta_{2} t+\beta_{3} t^{3 / 2}+O\left(t^{2}\right),
$$

where $\beta_{i}, i=0,1,2,3$ satisfy the equations in Appendix C. Solving these equations gives

$$
\begin{aligned}
& \beta_{0}=0.451723, \quad \beta_{1}=0.144914(r-q), \\
& \beta_{2}=-0.009801-0.041764(r+q)+0.014829(r-q)^{2}, \\
& \beta_{3}=-0.000618-0.002087(r-q)-0.015670\left(r^{2}-q^{2}\right)-0.001052(r-q)^{3} .
\end{aligned}
$$

\section{B Proof of Theorem 2}

The price of an American call option, $C(S, t)$, satisfies the PDE, boundary conditions and final conditions in equations $(12,13,14,15,16)$. By applying the transformation

$$
S=\frac{K^{2}}{\tilde{S}}, \quad C(S, t)=\frac{K}{\tilde{S}} P(\tilde{S}, t), \quad B^{c}(t)=\frac{K^{2}}{B^{p}(t)},
$$

we convert the problem of pricing an American call to a problem of pricing an American put with the same strike price but with a new interest rate, $\tilde{r}=q$, and a new dividend yield, $\tilde{q}=r$, i.e.,

$$
\begin{aligned}
& P_{t}+\frac{1}{2} \sigma^{2} \tilde{S}^{2} P_{\tilde{S} \tilde{S}}+(\tilde{r}-\tilde{q}) \tilde{S} P_{\tilde{S}}-\tilde{r} P=0, \quad \text { for } t<T, \quad \tilde{S}>B_{t}^{p} \text {, } \\
& P(\tilde{S}, t)=K-\tilde{S}, \quad P_{\tilde{S}}(\tilde{S}, t)=-1, \quad \text { for } t<T, \quad 0<\tilde{S} \leq B_{t}^{p} \text {, } \\
& P(\tilde{S}, T)=\max (K-\tilde{S}, 0), \\
& B_{T}^{p}=\left\{\begin{array}{ll}
K & \text { if } \quad \tilde{r} \geq \tilde{q} \\
\tilde{r} & \text { if } \quad \tilde{r}<\tilde{q}
\end{array} .\right.
\end{aligned}
$$

With the solution of the critical stock price, $B_{t}^{p}(q, r, \sigma, K, T)$, of an American put given by

Theorem 1, we can obtain the critical stock price for an American call by

$$
B_{t}^{c}(r, q, \sigma, K, T)=\frac{K^{2}}{B_{t}^{p}(q, r, \sigma, K, T)} .
$$


Note. The duality relation

$$
C(S, t ; r, q, \sigma, K, T)=\frac{S}{K} P\left(\frac{K^{2}}{S}, t ; q, r, \sigma, K, T\right)=S K P\left(\frac{1}{S}, t ; q, r, \sigma, \frac{1}{K}, T\right)
$$

is also called put-call symmetry. It was first discovered by Grabbe (1983) in the case of foreign-exchange options, where it has a natural interpretation. Building on the earlier work of Grabbe (1983), McDonald and Schroder (1990, 1998) recognized the relationship for American options in the binomial model. A review is offered by Carr and Chesney (1996). To the best of our knowledge, a clear proof in the PDE framework has not been offered before in the literature.

\section{Equations for $\beta_{i}, i=0,1,2,3$}

The equation to determine $\beta_{0}$ is

$$
-2 \beta_{0}^{2}=\frac{e^{-\beta_{0}^{2}}}{\sqrt{\pi}} \beta_{0}-\frac{2}{\sqrt{\pi}} \int_{-\infty}^{-\beta_{0}} e^{-z^{2}} d z\left(1+\beta_{0}^{2}\right)+\int_{0}^{1} \frac{1}{\sqrt{\pi z}} e^{-\frac{z \beta_{0}^{2}}{(1+\sqrt{1-z})^{2}}} \frac{\beta_{0}^{3} z}{(1+\sqrt{1-z})^{2}} d z .
$$

The equation to determine $\beta_{1}$ is

$$
\begin{array}{r}
-6 \beta_{0} \beta_{1}=-\frac{e^{-\beta_{0}^{2}}}{\sqrt{\pi}}\left(2 r-2 q-3 \beta_{1}\right)-\frac{1}{\sqrt{\pi}} \int_{-\infty}^{-\beta_{0}} e^{-z^{2}} d z\left(4 \beta_{0} \beta_{1}-3 \beta_{0} r+3 \beta_{0} q\right) \\
+\int_{0}^{1} \frac{1}{\sqrt{\pi z}} e^{-\frac{z \beta_{0}^{2}}{(1+\sqrt{1-z})^{2}}}\left[\beta_{0}^{4}\left(r-q-2 \beta_{1}\right) \frac{(1-\sqrt{1-z})^{2}}{1+\sqrt{1-z}}\right. \\
\left.+3 \beta_{0}^{2} \beta_{1} \frac{\sqrt{1-z}-1+z}{1+\sqrt{1-z}}+\beta_{0}^{2} \beta_{1}\right] d z .
\end{array}
$$

The equation to determine $\beta_{2}$ is

$$
\begin{aligned}
& -\frac{4}{3}\left(\beta_{0}^{4}-6 \beta_{0}^{2} \beta_{1}+3 \beta_{1}^{2}+6 \beta_{0} \beta_{2}\right) \\
& =\frac{e^{-\beta_{0}^{2}}}{12 \sqrt{\pi}}\left[8 \beta_{0}^{3}+\left(5+18 r-30 q-3 r^{2}+6 r q-3 q^{2}\right) \beta_{0}\right. \\
& \left.\quad-12(4-r+q) \beta_{0} \beta_{1}-12 \beta_{0} \beta_{1}^{2}+36 \beta_{2}\right] \\
& -\frac{1}{3 \sqrt{\pi}} \int_{-\infty}^{-\beta_{0}} e^{-z^{2}} d z\left[4 \beta_{0}^{4}-6 \beta_{0}^{2}\left(-1-r+2 q+3 \beta_{1}\right)+3\left(-r-q+r^{2}-2 r q+q^{2}\right)\right. \\
& \left.\quad+3(-2-3 r+3 q) \beta_{1}+6 \beta_{1}^{2}+12 \beta_{0} \beta_{2}\right] \\
& +\int_{0}^{1} \frac{1}{\sqrt{\pi z}} e^{-\frac{z \beta_{0}^{2}}{(1+\sqrt{1-z})^{2}}}\left[\frac { 1 } { 3 } \beta _ { 0 } \left(3 \sqrt{1-z}\left(-\beta_{0}^{2}+\beta_{1}\right)\right.\right.
\end{aligned}
$$




$$
\begin{aligned}
& \left(\beta_{1}-\frac{1}{z}(-2+2 \sqrt{1-z}+z)\left(2 \sqrt{1-z} \beta_{1}+(-1+\sqrt{1-z}) \beta_{0}^{2}\left(1-r+q+2 \beta_{1}\right)\right)\right) \\
& +\frac{1}{z}(-1+z)(-2+2 \sqrt{1-z}+z) \beta_{0}\left(2 \beta_{0}^{3}-6 \beta_{0} \beta_{1}+3 \beta_{2}\right) \\
& +3\left(-\beta_{1}\left(-2 \sqrt{1-z} \beta_{1}-(-1+\sqrt{1-z}) \beta_{0}^{2}\left(1-r+q+2 \beta_{1}\right)\right)\right. \\
& -\frac{1}{z}\left(-2+2(1-z)^{3 / 2}+z\right) \beta_{0} \beta_{2}+\frac{1}{z}\left((-2+2 \sqrt{1-z}+z) \beta_{0}(\right. \\
& -2(-1+\sqrt{1-z}) \sqrt{1-z} \beta_{0} \beta_{1}\left(1-r+q+2 \beta_{1}\right)+3(-1+z) \beta_{2} \\
& -\frac{1}{2} \beta_{0}\left((-1+\sqrt{1-z})^{2} \beta_{0}^{2}\left(1-r+q+2 \beta_{1}\right)^{2}+2\left(-r z-\frac{z}{4}\left(1-r+q+2 \beta_{1}\right)^{2}\right.\right. \\
& \left.\left.\left.\left.\left.\left.\left.-\frac{2}{z}\left(2-2 \sqrt{1-z}+(-2+\sqrt{1-z}) z+z^{2}\right) \beta_{0} \beta_{2}\right)\right)\right)\right)\right)\right)\right] d z .
\end{aligned}
$$

The equation to determine $\beta_{3}$ is

$$
\begin{aligned}
& \frac{2}{3}\left(\beta_{0}^{5}-10 \beta_{0}^{3} \beta_{1}+15 \beta_{0}^{2} \beta_{2}-15 \beta_{1} \beta_{2}+15 \beta_{0}\left(\beta_{1}^{2}-\beta_{3}\right)\right) \\
& =\frac{e^{-\beta_{0}^{2}}}{12 \sqrt{\pi}}\left[-4 \beta_{0}^{4}+\left(5+9 q^{2}+6 r+9 r^{2}-6 q(7+3 r)\right) \beta_{1}+12(-3+q-r) \beta_{1}^{2}\right. \\
& +4 \beta_{1}^{3}+\beta_{0}^{2}\left(-5+q^{3}-3 q^{2}(-1+r)-11 r+3 r^{2}-r^{3}+q\left(23-6 r+3 r^{2}\right)\right. \\
& \left.+2\left(19+3 q^{2}-6 q(-1+r)-6 r+3 r^{2}\right) \beta_{1}+12(1+q-r) \beta_{1}^{2}+8 \beta_{1}^{3}\right) \\
& \left.\left.-12 \beta_{0}\left(4+q-r+2 \beta_{1}\right) \beta_{2}+2\left(q+q^{3}+3 q r^{2}-3 q^{2}(2+r)-r\left(1-6 r+r^{2}\right) 18 \beta_{3}\right)\right)\right] \\
& +\frac{1}{6 \sqrt{\pi}} \int_{-\infty}^{-\beta_{0}} e^{-z^{2}} d z\left[4 \beta_{0}^{5}-4 \beta_{0}^{3}\left(-2+5 q-2 r+8 \beta_{1}\right)+36 \beta_{0}^{2} \beta_{2}\right. \\
& -6\left(-2+3 q-3 r+4 \beta_{1}\right) \beta_{2} \\
& \left.+3 \beta_{0}\left(-4 q+5 q^{2}-4 q r-r^{2}+8(-1+2 q-r) \beta_{1}+12 \beta_{1}^{2}-8 \beta_{3}\right)\right] \\
& +\int_{0}^{1} \frac{1}{\sqrt{\pi z}} e^{-\frac{z \beta_{0}^{2}}{(1+\sqrt{1-z})^{2}}}\left[\frac{1}{3}(-1+z) \beta_{0}\right. \\
& \left(\beta_{1}-\frac{1}{z}(-2+2 \sqrt{1-z}+z)\left(2 \sqrt{1-z} \beta_{1}+(-1+\sqrt{1-z}) \beta_{0}^{2}\left(1+q-r+2 \beta_{1}\right)\right)\right) \\
& \left(-2 \beta_{0}^{3}+6 \beta_{0} \beta_{1}-3 \beta_{2}\right) \\
& +\sqrt{1-z}\left(-\beta_{0}^{2}+\beta_{1}\right)\left(-\beta_{1}\left(-2 \sqrt{1-z} \beta_{1}-(-1+\sqrt{1-z}) \beta_{0}^{2}\left(1+q-r+2 \beta_{1}\right)\right)\right. \\
& -\frac{1}{z}\left(-2+2(1-z)^{3 / 2}+z\right) \beta_{0} \beta_{2} \\
& +\frac{1}{z}\left(( - 2 + 2 \sqrt { 1 - z } + z ) \beta _ { 0 } \left(-2(-1+\sqrt{1-z}) \sqrt{1-z} \beta_{0} \beta_{1}\left(1+q-r+2 \beta_{1}\right)\right.\right. \\
& +3(-1+z) \beta_{2}-\frac{1}{2} \beta_{0}\left((-1+\sqrt{1-z})^{2} \beta_{0}^{2}\left(1+q-r+2 \beta_{1}\right)^{2}\right. \\
& \left.\left.\left.\left.+2\left(-r z-\frac{z}{4}\left(1+q-r+2 \beta_{1}\right)^{2}-\frac{2}{z}\left(2-2 \sqrt{1-z}+(-2+\sqrt{1-z}) z+z^{2}\right) \beta_{0} \beta_{2}\right)\right)\right)\right)\right)
\end{aligned}
$$




$$
\begin{aligned}
& +\frac{1}{3 z}(1-z)^{3 / 2}(-2+2 \sqrt{1-z}+z) \beta_{0}^{2}\left(\beta_{0}^{4}-6 \beta_{0}^{2} \beta_{1}+3 \beta_{1}^{2}+6 \beta_{0} \beta_{2}-3 \beta_{3}\right) \\
& +\beta_{0}\left(\frac{1}{z}\left(-2+2(1-z)^{3 / 2}+z\right)\left(-2 \sqrt{1-z} \beta_{1}-(-1+\sqrt{1-z}) \beta_{0}^{2}\left(1+q-r+2 \beta_{1}\right)\right) \beta_{2}\right. \\
& -\beta_{1}\left(-2(-1+\sqrt{1-z}) \sqrt{1-z} \beta_{0} \beta_{1}\left(1+q-r+2 \beta_{1}\right)\right. \\
& +3(-1+z) \beta_{2}-\frac{1}{2} \beta_{0}\left((-1+\sqrt{1-z})^{2} \beta_{0}^{2}\left(1+q-r+2 \beta_{1}\right)^{2}\right. \\
& \left.\left.+2\left(-r z-\frac{z}{4}\left(1+q-r+2 \beta_{1}\right)^{2}-\frac{2}{z}\left(2-2 \sqrt{1-z}+(-2+\sqrt{1-z}) z+z^{2}\right) \beta_{0} \beta_{2}\right)\right)\right) \\
& -(-3+2 z) \beta_{0} \beta_{3}+\frac{1}{z}\left(( - 2 + 2 \sqrt { 1 - z } + z ) \beta _ { 0 } \left(3(-1+\sqrt{1-z})(-1+z) \beta_{0}\right.\right. \\
& \left(1+q-r+2 \beta_{1}\right) \beta_{2}-\sqrt{1-z} \beta_{1}\left((-1+\sqrt{1-z})^{2} \beta_{0}^{2}\left(1+q-r+2 \beta_{1}\right)^{2}\right. \\
& \left.+2\left(-r z-\frac{z}{4}\left(1+q-r+2 \beta_{1}\right)^{2}-\frac{2}{z}\left(2-2 \sqrt{1-z}+(-2+\sqrt{1-z}) z+z^{2}\right) \beta_{0} \beta_{2}\right)\right) \\
& -4(1-z)^{3 / 2} \beta_{3}-\frac{1}{6} \beta_{0}\left(4(-1+\sqrt{1-z}) \beta_{0}\left(1+q-r+2 \beta_{1}\right)\right. \\
& \left(-r z-\frac{z}{4}\left(1+q-r+2 \beta_{1}\right)^{2}-\frac{2}{z}\left(2-2 \sqrt{1-z}+(-2+\sqrt{1-z}) z+z^{2}\right) \beta_{0} \beta_{2}\right) \\
& +(-1+\sqrt{1-z}) \beta_{0}\left(1+q-r+2 \beta_{1}\right)\left((-1+\sqrt{1-z})^{2} \beta_{0}^{2}\left(1+q-r+2 \beta_{1}\right)^{2}\right. \\
& \left.+2\left(-r z-\frac{z}{4}\left(1+q-r+2 \beta_{1}\right)^{2}-\frac{2}{z}\left(2-2 \sqrt{1-z}+(-2+\sqrt{1-z}) z+z^{2}\right) \beta_{0} \beta_{2}\right)\right) \\
& \left.\left.\left.\left.\left.+6\left(\left(-1+(1-z)^{3 / 2}\right)\left(1+q-r+2 \beta_{1}\right) \beta_{2}-2(-1+\sqrt{1-z})(-2+z) \beta_{0} \beta_{3}\right)\right)\right)\right)\right)\right] d z .
\end{aligned}
$$




\section{References}

[1] Barone-Adesi, G., and R. E. Whaley (1987): Efficient Analytic Approximation of American Option Values, J. Finance 42, 301-320.

[2] Black, F., and M. Scholes (1973): The Pricing of Options and Corporate Liabilities, J. Polit. Econ. 81, 637-654.

[3] Brennan, M. J., and E. S. Schwartz (1977): The Valuation of American Options, J. Finance 32, 449-462.

[4] Broadie, M., and J. Detemple (1996): American Option Valuation: New bounds, Approximations, and a Comparison of Existing Methods, Rev. Financ. Stud. 9, 1211-1250.

[5] Bunch, D. S., and H. Johnson (1992): A Simple and Numerically Efficient Valuation Method for American Puts Using a Modified Geske-Johnson Approach, J. Finance 47, 809-816.

[6] Bunch, D. S., and H. Johnson (2000): The American Put Option and Its Critical Stock Price, J. Finance 55, 2333-2356.

[7] Cannon, J. R. (1984): The one-dimensional heat equation, Encyclopedia of mathematics and its application, Vol. 23, Addison-Wesley Publishing Company.

[8] Carr, P. (1998): Randomization and the American Put, Rev. Financ. Stud. 11, 597-626.

[9] Carr, P., M. Chesney (1996): American Put Call Symmetry, Working paper, Morgan Stanley New York and Groupe H.E.C. France.

[10] Carr, P., R. Jarrow, and R. Myneni (1992): Alternative Characterizations of American Put Otions, Math. Finance 2, 87-106.

[11] Chen, X.-F., J. Chadam (2007): A Mathematical Analysis for the Optimal Exercise Boundary of American Put Options, SIAM J. Math. Analysis 38, 1613-1641. 
[12] Cox, J., S. Ross, and M. Rubinstein (1979): Option Pricing: A Simplified Approach, J. Financ. Economics 7, 229-263.

[13] Evans, J. D., R. A. Kuske, and J. B. Keller (2002): American Options on Assets with Divdidends Near Expiry, Math. Finance 12, 219-237.

[14] Geske, R., and H. Johnson (1984): The American Put Option Valued Analytically, J. Finance 39, 1511-1524.

[15] Grabbe, J. (1983): The Pricing of Call and Put Options on Foreign Exchange, J. International Money and Finance 2, 239-253.

[16] Huang, J.-Z., M. G. Subrahmanyam, and G. G. Yu (1996): Pricing and Hedging American Options: A Recursive Integration Method, Rev. Financ. Stud. 9, 277-300.

[17] Jacka, S. D. (1991): Optimal Stopping and the American Put, Math. Finance 1, 1-14.

[18] Johnson, H. E. (1983): An Analytic Approximation for the American Put Price, J. Financ. Quant. Analysis 18, 141-148.

[19] Ju, N.-J. (1998): Pricing an American Option by Approximating Its Early Exercise Boundary as a Multipiece Exponential Function, Rev. Financ. Stud. 11, 627-646.

[20] Kim, I. J. (1990): The Analytic Valuation of American Options, Rev. Financ. Stud. $3,547-572$.

[21] Kolodner, I. I. (1956): Free Boundary Problem for the Heat Equation with Applications to Problems of Change of Phase, Communications in Pure and Applied Mathematics 9, 1-31.

[22] Kuske, R. A., and J. B. Keller (1998): Optimal Exercise Boundary for an American Put Option, Appl. Math. Finance 5, 107-116.

[23] Longstaff, F. A., and E. S. Schwartz (2001): Valuing American Options by Simulation: A Simple Least-squares Approach, Rev. Financ. Stud. 14, 113-147. 
[24] MacMillan, L. W. (1986): Analytic Approximation for the American Put Option, Advances in Futures and Options Research 1, 119-139.

[25] McDonald, R. L., and M. D. Schroder (1990): A Parity Result for American Options, Working paper, Northwestern University.

[26] McDonald, R. L., and Mark D. Schroder (1998): A Parity Result for American Options, J. Computational Finance 1, 5-13.

[27] McKean, H. P., Jr. (1965): Appendix: A Free Boundary Problem for the Heat Equation Arising from a Problem in Mathematical Economics, Industrial Management Review $6,32-39$.

[28] Merton, R. (1973): The Theory of Rational Option Pricing, Bell Journal of Economics 4, 141-183.

[29] Parkinson, M. (1977): Option Pricing: the American Put, J. Business 50, 21-36.

[30] Stamicar, R., D. Ševčovič, and J. Chadam (1999): The Early Exercise Boundary for the American Put Near Expiry: Numerical Approximation, Canadian Applied Mathematics Quarterly 7, 427-444.

[31] Yu, G. G. (1993): Essays on the Valuation of American Options, Ph.D. dissertation, New York University.

[32] Zhang, J. E. (2003): Pricing Continuously Sampled Asian Options with Perturbation Method, J. Futures Markets 23, 535-560.

[33] Zhu, S.-P. (2006): An Exact and Explicit Solution for the Valuation of American Put Options, Quant. Finance 6, 229-242. 
Table 1: The critical stock price, $B_{t}^{p}$, of an American put option with a strike price $K=100$ - Convergence of the numerical values computed by solving the integral equation.

$$
\begin{aligned}
K-B_{t}^{p}= & K e^{-r(T-t)} N\left(-d_{2}\left(B_{t}^{p}, K, T-t\right)\right)-B_{t}^{p} e^{-q(T-t)} N\left(-d_{1}\left(B_{t}^{p}, K, T-t\right)\right) \\
& +\int_{t}^{T}\left[r K e^{-r s} N\left(-d_{2}\left(B_{t}^{p}, B_{s}^{p}, s\right)\right)-q B_{t}^{p} e^{-q s} N\left(-d_{1}\left(B_{t}^{p}, B_{s}^{p}, s\right)\right)\right] d s .
\end{aligned}
$$

Parameters: $r=0.05, q=0$ and $\sigma=0.3$

\begin{tabular}{|cccccccc|}
\hline S $t$ & $1 / 52$ & $1 / 104$ & $1 / 208$ & $1 / 416$ & $1 / 832$ & $1 / 1664$ & $1 / 3328$ \\
\hline 0 & 100.0000 & 100.0000 & 100.0000 & 100.0000 & 100.0000 & 100.0000 & 100.0000 \\
$1 / 52$ & 91.4974 & 91.3575 & 91.3407 & 91.3356 & 91.3342 & 91.3339 & 91.3338 \\
$2 / 52$ & 88.7423 & 88.7417 & 88.7417 & 88.7423 & 88.7427 & 88.7430 & 88.7431 \\
$3 / 52$ & 86.9467 & 86.9524 & 86.9556 & 86.9572 & 86.9579 & 86.9582 & 86.9583 \\
$4 / 52$ & 85.5495 & 85.5587 & 85.5627 & 85.5645 & 85.5652 & 85.5655 & 85.5657 \\
$5 / 52$ & 84.3953 & 84.4053 & 84.4095 & 84.4112 & 84.4119 & 84.4122 & 84.4124 \\
$6 / 52$ & 83.4060 & 83.4160 & 83.4201 & 83.4218 & 83.4225 & 83.4228 & 83.4229 \\
$7 / 52$ & 82.5372 & 82.5470 & 82.5510 & 82.5526 & 82.5533 & 82.5535 & 82.5536 \\
$8 / 52$ & 81.7612 & 81.7706 & 81.7744 & 81.7760 & 81.7766 & 81.7768 & 81.7769 \\
$9 / 52$ & 81.0589 & 81.0680 & 81.0716 & 81.0731 & 81.0736 & 81.0739 & 81.0740 \\
$10 / 52$ & 80.4169 & 80.4256 & 80.4291 & 80.4305 & 80.4310 & 80.4312 & 80.4313 \\
$11 / 52$ & 79.8253 & 79.8336 & 79.8369 & 79.8382 & 79.8387 & 79.8390 & 79.8390 \\
$12 / 52$ & 79.2764 & 79.2844 & 79.2875 & 79.2888 & 79.2893 & 79.2894 & 79.2895 \\
$13 / 52$ & 78.7643 & 78.7719 & 78.7749 & 78.7761 & 78.7766 & 78.7768 & 78.7768 \\
$14 / 52$ & 78.2842 & 78.2915 & 78.2944 & 78.2955 & 78.2960 & 78.2962 & 78.2962 \\
$15 / 52$ & 77.8323 & 77.8393 & 77.8421 & 77.8432 & 77.8436 & 77.8438 & 77.8438 \\
$16 / 52$ & 77.4054 & 77.4121 & 77.4148 & 77.4158 & 77.4162 & 77.4164 & 77.4165 \\
$17 / 52$ & 77.0009 & 77.0073 & 77.0099 & 77.0109 & 77.0113 & 77.0114 & 77.0115 \\
\hline
\end{tabular}


Table 2: The price, $P(S, t)$, of an American put option with a strike price $K=100$ at time $t$ - Convergence of the numerical values computed by solving the integral equation where $B_{t}^{p}$ is given by Table 1 .

$$
\begin{aligned}
P(S, t)= & K e^{-r(T-t)} N\left(-d_{2}(S, K, T-t)\right)-S e^{-q(T-t)} N\left(-d_{1}(S, K, T-t)\right) \\
& +\int_{t}^{T}\left[r K e^{-r s} N\left(-d_{2}\left(S, B_{s}^{p}, s\right)\right)-q S e^{-q s} N\left(-d_{1}\left(S, B_{s}^{p}, s\right)\right)\right] d s
\end{aligned}
$$

Parameters: $r=0.05, q=0$ and $\tau \doteq T-t=1 / 12$

\begin{tabular}{|cccccc|}
\hline$\Delta t / \tau$ & $1 / 20$ & $1 / 40$ & $1 / 80$ & $1 / 160$ & $1 / 320$ \\
\hline$(9, \sigma)$ & & & & & \\
$(95,0.2)$ & 9.9905 & 9.9950 & 9.9974 & 9.9987 & 10.0000 \\
$(100,0.2)$ & 5.3572 & 5.3567 & 5.3565 & 5.3564 & 5.3564 \\
$(105,0.2)$ & 0.5808 & 0.5804 & 0.5802 & 0.5801 & 0.5801 \\
$(110,0.2)$ & 0.1054 & 0.1053 & 0.1052 & 0.1052 & 0.1052 \\
$(90,0.3)$ & 10.2316 & 10.2312 & 10.2310 & 10.2309 & 10.2309 \\
$(95,0.3)$ & 6.2098 & 6.2091 & 6.2088 & 6.2087 & 6.2087 \\
$(100,0.3)$ & 3.2726 & 3.2718 & 3.2715 & 3.2714 & 3.2713 \\
$(105,0.3)$ & 1.4763 & 1.4757 & 1.4755 & 1.4754 & 1.4754 \\
$(110,0.3)$ & 0.5678 & 0.5674 & 0.5673 & 0.5672 & 0.5672 \\
$(90,0.4)$ & 10.8325 & 10.8318 & 10.8316 & 10.8315 & 10.8314 \\
$(95,0.4)$ & 7.1948 & 7.1940 & 7.1937 & 7.1936 & 7.1935 \\
$(100,0.4)$ & 4.4183 & 4.4174 & 4.4171 & 4.4170 & 4.4169 \\
$(105,0.4)$ & 2.5018 & 2.5011 & 2.5008 & 2.5007 & 2.5007 \\
$(110,0.4)$ & 1.3070 & 1.3065 & 1.3063 & 1.3063 & 1.3062 \\
$(90,0.5)$ & 11.6092 & 11.6085 & 11.6081 & 11.6080 & 11.6080 \\
$(95,0.5)$ & 8.2325 & 8.2316 & 8.2313 & 8.2312 & 8.2311 \\
$(100,0.5)$ & 5.5638 & 5.5630 & 5.5626 & 5.5625 & 5.5625 \\
$(105,0.5)$ & 3.5842 & 3.5834 & 3.5831 & 3.5830 & 3.5830 \\
$(110,0.5)$ & 2.2039 & 2.2033 & 2.2031 & 2.2030 & 2.2030 \\
\hline
\end{tabular}


Table 3: The critical stock price, $B_{t}^{p}$, of an American put option with a strike price $K=100$ - Accuracy of the present truncated analytical formulas for the case $r>q$.

$$
B_{t}^{p}=K e^{-\sqrt{2 \sigma^{2}(T-t) u(\xi)}}, \quad \xi=\ln \sqrt{8 \pi(r-q)^{2}(T-t) / \sigma^{2}},
$$

TA1 : $u(\xi)=-\xi$

TA2 : $u(\xi)=-\xi-\frac{1}{2 \xi}$,

TA3 : $u(\xi)=-\xi-\frac{1}{2 \xi}+\frac{1}{8 \xi^{2}}$,

TA4 : $u(\xi)=-\xi-\frac{1}{2 \xi}+\frac{1}{8 \xi^{2}}+\frac{11}{24 \xi^{3}}$.

Parameters: $r=0.05, q=0$ and $\sigma=0.3$

\begin{tabular}{|cccccc|}
\hline$T-t$ & Highly Accurate & TA1 & TA2 & TA3 & TA4 \\
\hline 0 & 100.0000 & 100.0000 & 100.0000 & 100.0000 & 100.0000 \\
$1 / 52$ & 91.3338 & 91.7250 & 91.3105 & 91.2638 & 91.3433 \\
$2 / 52$ & 88.7431 & 89.4132 & 88.6789 & 88.5817 & 88.7797 \\
$3 / 52$ & 86.9583 & 87.8849 & 86.8398 & 86.6860 & 87.0404 \\
$4 / 52$ & 85.5657 & 86.7374 & 85.3807 & 85.1640 & 85.7151 \\
$1 / 12$ & 85.1584 & 86.4111 & 84.9496 & 84.7105 & 85.3369 \\
$5 / 52$ & 84.4124 & 85.8224 & 84.1493 & 83.8635 & 84.6555 \\
$6 / 52$ & 83.4229 & 85.0670 & 83.0706 & 82.7091 & 83.7910 \\
$7 / 52$ & 82.5536 & 84.4289 & 82.1009 & 81.6573 & 83.0839 \\
$8 / 52$ & 81.7769 & 83.8816 & 81.2128 & 80.6802 & 82.5135 \\
$1 / 6$ & 81.3009 & 83.5580 & 80.6564 & 80.0608 & 82.2039 \\
$9 / 52$ & 81.0740 & 83.4070 & 80.3873 & 79.7590 & 82.0696 \\
$10 / 52$ & 80.4313 & 82.9922 & 79.6109 & 78.8798 & 81.7490 \\
$11 / 52$ & 79.8390 & 82.6276 & 78.8736 & 78.0325 & 81.5547 \\
$12 / 52$ & 79.2895 & 82.3060 & 78.1677 & 77.2091 & 81.4951 \\
$1 / 4$ & 78.7768 & 82.0219 & 77.4870 & 76.4032 & 81.5853 \\
$14 / 52$ & 78.2962 & 81.7706 & 76.8266 & 75.6098 & 81.8486 \\
$15 / 52$ & 77.8438 & 81.5485 & 76.1825 & 74.8246 & 82.3196 \\
$16 / 52$ & 77.4165 & 81.3528 & 75.5512 & 74.0438 & 83.0513 \\
$17 / 52$ & 77.0115 & 81.1807 & 74.9298 & 73.2643 & 84.1288 \\
$1 / 3$ & 76.8810 & 81.1283 & 74.7245 & 73.0043 & 84.5875 \\
\hline
\end{tabular}

The "Highly Accurate" value is the converged numerical result obtained by numerically solving the following integral equation:

$$
\begin{aligned}
K-B_{t}^{p}= & K e^{-r(T-t)} N\left(-d_{2}\left(B_{t}^{p}, K, T-t\right)\right)-B_{t}^{p} e^{-q(T-t)} N\left(-d_{1}\left(B_{t}^{p}, K, T-t\right)\right) \\
& +\int_{t}^{T}\left[r K e^{-r s} N\left(-d_{2}\left(B_{t}^{p}, B_{s}^{p}, s\right)\right)-q B_{t}^{p} e^{-q s} N\left(-d_{1}\left(B_{t}^{p}, B_{s}^{p}, s\right)\right)\right] d s .
\end{aligned}
$$


Table 4: The price, $P(S, t)$, of an American put option with a strike price $K=100$ at time $t$ - Accuracy of the present truncated analytical formulas for the case $r>q$.

$$
\begin{array}{ll}
P(S, t)= & K e^{-r(T-t)} N\left(-d_{2}(S, K, T-t)\right)-S e^{-q(T-t)} N\left(-d_{1}(S, K, T-t)\right) \\
& +\int_{t}^{T}\left[r K e^{-r s} N\left(-d_{2}\left(S, B_{s}^{p}, s\right)\right)-q S e^{-q s} N\left(-d_{1}\left(S, B_{s}^{p}, s\right)\right)\right] d s, \\
\text { where } \quad & B_{t}^{p}=K e^{-\sqrt{2 \sigma^{2}(T-t) u(\xi)}}, \quad \xi=\ln \sqrt{8 \pi(r-q)^{2}(T-t) / \sigma^{2}}, \\
& \text { TA1 }: u(\xi)=-\xi, \\
& \text { TA2 }: u(\xi)=-\xi-\frac{1}{2 \xi}, \\
& \text { TA3 }: u(\xi)=-\xi-\frac{1}{2 \xi}+\frac{1}{8 \xi^{2}}, \\
& \text { TA4 }: u(\xi)=-\xi-\frac{1}{2 \xi}+\frac{1}{8 \xi^{2}}+\frac{11}{24 \xi^{3}} .
\end{array}
$$

Parameters: $r=0.05, q=0$ and $\tau \doteq T-t=1 / 12$

\begin{tabular}{|cccccc|}
\hline$(S, \sigma)$ & Highly Accurate & TA1 & TA2 & TA3 & TA4 \\
\hline$(90,0.2)$ & 10.0000 & 10.0000 & 10.0000 & 10.0000 & 10.0000 \\
$(95,0.2)$ & 5.3564 & 5.3770 & 5.3547 & 5.3516 & 5.3615 \\
$(100,0.2)$ & 2.1269 & 2.1310 & 2.1267 & 2.1262 & 2.1276 \\
$(105,0.2)$ & 0.5801 & 0.5807 & 0.5801 & 0.5800 & 0.5802 \\
$(110,0.2)$ & 0.1052 & 0.1053 & 0.1052 & 0.1052 & 0.1052 \\
$(90,0.3)$ & 10.2309 & 10.2484 & 10.2291 & 10.2268 & 10.2321 \\
$(95,0.3)$ & 6.2087 & 6.2154 & 6.2081 & 6.2072 & 6.2090 \\
$(100,0.3)$ & 3.2713 & 3.2737 & 3.2712 & 3.2709 & 3.2714 \\
$(105,0.3)$ & 1.4754 & 1.4761 & 1.4753 & 1.4752 & 1.4754 \\
$(110,0.3)$ & 0.5672 & 0.5674 & 0.5672 & 0.5672 & 0.5672 \\
$(90,0.4)$ & 10.8314 & 10.8386 & 10.8301 & 10.8292 & 10.8308 \\
$(95,0.4)$ & 7.1935 & 7.1969 & 7.1929 & 7.1925 & 7.1932 \\
$(100,0.4)$ & 4.4169 & 4.4185 & 4.4167 & 4.4165 & 4.4168 \\
$(105,0.4)$ & 2.5007 & 2.5013 & 2.5006 & 2.5005 & 2.5006 \\
$(110,0.4)$ & 1.3062 & 1.3065 & 1.3062 & 1.3061 & 1.3062 \\
$(90,0.5)$ & 11.6080 & 11.6117 & 11.6067 & 11.6062 & 11.6070 \\
$(95,0.5)$ & 8.2311 & 8.2332 & 8.2304 & 8.2302 & 8.2305 \\
$(100,0.5)$ & 5.5625 & 5.5636 & 5.5621 & 5.5620 & 5.5622 \\
$(105,0.5)$ & 3.5830 & 3.5835 & 3.5828 & 3.5827 & 3.5828 \\
$(110,0.5)$ & 2.2030 & 2.2032 & 2.2029 & 2.2028 & 2.2029 \\
\hline RMSE & & 0.0067 & 0.0007 & 0.0016 & 0.0012 \\
MAE & & 0.0206 & 0.0018 & 0.0048 & 0.0051 \\
\hline
\end{tabular}

The "Highly Accurate" value is obtained by numerically computing the integration with the highly accurate critical stock prices presented in Table 3. RMSE is the root of the mean squared errors. MAE is the maximum absolute error. 
Table 5: The critical stock price, $B_{t}^{p}$, of an American put option with a strike price $K=100$ - Accuracy of the present truncated analytical formulas for the case $r=q$.

$$
B_{t}^{p}=K e^{-\sqrt{2 \sigma^{2}(T-t) v(\eta)}}, \quad \eta=\ln [4 \sqrt{\pi} r(T-t)],
$$

TA1 : $v(\eta)=-\eta$,

TA2 : $v(\eta)=-\eta-\frac{1}{2} \ln (-\eta)$,

TA3 : $v(\eta)=-\eta-\frac{1}{2} \ln (-\eta)-\frac{1}{4 \eta} \ln (-\eta)$,

TA4 : $\quad v(\eta)=-\eta-\frac{1}{2} \ln (-\eta)-\frac{1}{4 \eta} \ln (-\eta)-\frac{1-\frac{5}{4 \sqrt{2 \pi}}}{\eta}$.

Parameters: $r=0.05, q=0.05$ and $\sigma=0.3$

\begin{tabular}{|cccccc|}
\hline$T-t$ & Highly Accurate & TA1 & TA2 & TA3 & TA4 \\
\hline 0 & 100.0000 & 100.0000 & 100.0000 & 100.0000 & 100.0000 \\
$1 / 52$ & 88.2254 & 87.6863 & 88.6604 & 88.5583 & 88.4323 \\
$2 / 52$ & 84.7617 & 84.1608 & 85.4591 & 85.3005 & 85.0857 \\
$3 / 52$ & 82.3911 & 81.7929 & 83.3107 & 83.1055 & 82.8084 \\
$4 / 52$ & 80.5499 & 79.9855 & 81.6695 & 81.4233 & 81.0467 \\
$1 / 12$ & 80.0129 & 79.4655 & 81.1967 & 80.9378 & 80.5350 \\
$5 / 52$ & 79.0311 & 78.5192 & 80.3353 & 80.0520 & 79.5972 \\
$6 / 52$ & 77.7323 & 77.2856 & 79.2097 & 78.8923 & 78.3598 \\
$7 / 52$ & 76.5946 & 76.2225 & 78.2362 & 77.8871 & 77.2769 \\
$8 / 52$ & 75.5807 & 75.2903 & 77.3790 & 77.0002 & 76.3121 \\
$1 / 6$ & 74.9605 & 74.7280 & 76.8599 & 76.4623 & 75.7220 \\
$9 / 52$ & 74.6651 & 74.4622 & 76.6139 & 76.2072 & 75.4407 \\
$10 / 52$ & 73.8299 & 73.7193 & 75.9236 & 75.4906 & 74.6450 \\
$11 / 52$ & 73.0617 & 73.0473 & 75.2955 & 74.8377 & 73.9122 \\
$12 / 52$ & 72.3503 & 72.4357 & 74.7198 & 74.2387 & 73.2322 \\
$1 / 4$ & 71.6877 & 71.8759 & 74.1889 & 73.6858 & 72.5975 \\
$14 / 52$ & 71.0676 & 71.3613 & 73.6969 & 73.1731 & 72.0017 \\
$15 / 52$ & 70.4848 & 70.8865 & 73.2387 & 72.6955 & 71.4400 \\
$16 / 52$ & 69.9351 & 70.4470 & 72.8105 & 72.2491 & 70.9081 \\
$17 / 52$ & 69.4149 & 70.0390 & 72.4088 & 71.8305 & 70.4027 \\
$1 / 3$ & 69.2474 & 69.9095 & 72.2803 & 71.6966 & 70.2396 \\
\hline
\end{tabular}

The "Highly Accurate" value is the converged numerical result obtained by numerically solving the following integral equation:

$$
\begin{aligned}
K-B_{t}^{p}= & K e^{-r(T-t)} N\left(-d_{2}\left(B_{t}^{p}, K, T-t\right)\right)-B_{t}^{p} e^{-q(T-t)} N\left(-d_{1}\left(B_{t}^{p}, K, T-t\right)\right) \\
& +\int_{t}^{T}\left[r K e^{-r s} N\left(-d_{2}\left(B_{t}^{p}, B_{s}^{p}, s\right)\right)-q B_{t}^{p} e^{-q s} N\left(-d_{1}\left(B_{t}^{p}, B_{s}^{p}, s\right)\right)\right] d s .
\end{aligned}
$$


Table 6: The price, $P(S, t)$, of an American put option with a strike price $K=100$ at time $t$ - Accuracy of the present truncated analytical formulas for the case $r=q$.

$$
\begin{array}{ll}
P(S, t)= & K e^{-r(T-t)} N\left(-d_{2}(S, K, T-t)\right)-S e^{-q(T-t)} N\left(-d_{1}(S, K, T-t)\right) \\
& +\int_{t}^{T}\left[r K e^{-r s} N\left(-d_{2}\left(S, B_{s}^{p}, s\right)\right)-q S e^{-q s} N\left(-d_{1}\left(S, B_{s}^{p}, s\right)\right)\right] d s, \\
\text { where } \quad & B_{t}^{p}=K e^{-\sqrt{2 \sigma^{2}(T-t) v(\eta)}}, \quad \eta=\ln [4 \sqrt{\pi} r(T-t)], \\
& \text { TA1 : } v(\eta)=-\eta, \\
& \text { TA2 : } v(\eta)=-\eta-\frac{1}{2} \ln (-\eta), \\
& \text { TA3 : } v(\eta)=-\eta-\frac{1}{2} \ln (-\eta)-\frac{1}{4 \eta} \ln (-\eta), \\
& \text { TA4 }: \quad v(\eta)=-\eta-\frac{1}{2} \ln (-\eta)-\frac{1}{4 \eta} \ln (-\eta)-\frac{1-\frac{5}{4 \sqrt{2 \pi}}}{\eta} .
\end{array}
$$

Parameters: $r=0.05, q=0.05$ and $\tau \doteq T-t=1 / 12$

\begin{tabular}{|cccccc|}
\hline$(S, \sigma)$ & Highly Accurate & TA1 & TA2 & TA3 & TA4 \\
\hline$(90,0.2)$ & 10.0535 & 10.0522 & 10.0554 & 10.0549 & 10.0543 \\
$(95,0.2)$ & 5.5603 & 5.5598 & 5.5607 & 5.5606 & 5.5604 \\
$(100,0.2)$ & 2.2947 & 2.2947 & 2.2949 & 2.2949 & 2.2948 \\
$(105,0.2)$ & 0.6537 & 0.6537 & 0.6537 & 0.6537 & 0.6537 \\
$(110,0.2)$ & 0.1242 & 0.1242 & 0.1242 & 0.1242 & 0.1242 \\
$(90,0.3)$ & 10.4167 & 10.4157 & 10.4178 & 10.4176 & 10.4172 \\
$(95,0.3)$ & 6.4202 & 6.4197 & 6.4205 & 6.4204 & 6.4203 \\
$(100,0.3)$ & 3.4415 & 3.4414 & 3.4417 & 3.4417 & 3.4416 \\
$(105,0.3)$ & 1.5812 & 1.5812 & 1.5812 & 1.5812 & 1.5812 \\
$(110,0.3)$ & 0.6197 & 0.6198 & 0.6198 & 0.6198 & 0.6198 \\
$(90,0.4)$ & 11.0410 & 11.0400 & 11.0418 & 11.0416 & 11.0413 \\
$(95,0.4)$ & 7.3994 & 7.3989 & 7.3997 & 7.3996 & 7.3995 \\
$(100,0.4)$ & 4.5875 & 4.5875 & 4.5878 & 4.5878 & 4.5877 \\
$(105,0.4)$ & 2.6239 & 2.6238 & 2.6240 & 2.6240 & 2.6239 \\
$(110,0.4)$ & 1.3851 & 1.3850 & 1.3851 & 1.3851 & 1.3850 \\
$(90,0.5)$ & 11.8191 & 11.8182 & 11.8199 & 11.8197 & 11.8194 \\
$(95,0.5)$ & 8.4307 & 8.4301 & 8.4310 & 8.4309 & 8.4308 \\
$(100,0.5)$ & 5.7326 & 5.7325 & 5.7330 & 5.7330 & 5.7329 \\
$(105,0.5)$ & 3.7164 & 3.7163 & 3.7165 & 3.7165 & 3.7165 \\
$(110,0.5)$ & 2.2998 & 2.2999 & 2.3000 & 2.3000 & 2.3000 \\
\hline RMSE & & 0.0005 & 0.0006 & 0.0005 & 0.0003 \\
MAE & & 0.0013 & 0.0019 & 0.0014 & 0.0008 \\
\hline
\end{tabular}

The "Highly Accurate" value is obtained by numerically computing the integration with the highly accurate critical stock prices presented in Table 5. RMSE is the root of the mean squared errors. MAE is the maximum absolute error. 
Table 7: The critical stock price, $B_{t}^{p}$, of an American put option with a strike price $K=100$ - Accuracy of the present truncated analytical formulas for the case $r<q$.

$$
\begin{aligned}
& B_{t}^{p}=\frac{r}{q} K e^{-2 \sqrt{\tau^{*}} w\left(\sqrt{\tau^{*}}\right)}, \quad \tau^{*}=\frac{1}{2} \sigma^{2}(T-t), \quad r^{*}=\frac{r}{\frac{1}{2} \sigma^{2}}, \quad q^{*}=\frac{q}{\frac{1}{2} \sigma^{2}}, \\
& \text { TA1 }: \quad w\left(\sqrt{\tau^{*}}\right)=\beta_{0}, \\
& \text { TA3 }: \quad w\left(\sqrt{\tau^{*}}\right)=\beta_{0}+\beta_{1} \sqrt{\tau^{*}}+\beta_{2} \tau^{*}, \quad \text { TA2 }: \quad w\left(\sqrt{\tau^{*}}\right)=\beta_{0}+\beta_{1} \sqrt{\tau^{*}}, \\
& \beta_{0}=0.451723, \quad \beta_{1}=0.144914\left(r^{*}-q^{*}\right), \\
& \beta_{2}=-0.009801-0.041764\left(r^{*}+q^{*}\right)+0.014829\left(r^{*}-q^{*}\right)^{2}, \\
& \beta_{3}=-0.000618-0.002087\left(r^{*}-q^{*}\right)-0.015670\left(r^{* 2}-q^{* 2}\right)-0.001052\left(r^{*}-q^{*}\right)^{3} .
\end{aligned}
$$

Parameters: $r=0.05, q=0.07$ and $\sigma=0.3$

\begin{tabular}{|cccccc|}
\hline$T-t$ & Highly Accurate & TA1 & TA2 & TA3 & TA4 \\
\hline 0 & 71.4286 & 71.4286 & 71.4286 & 71.4286 & 71.4286 \\
$1 / 52$ & 69.5641 & 69.5552 & 69.5630 & 69.5634 & 69.5634 \\
$2 / 52$ & 68.8106 & 68.7937 & 68.8090 & 68.8102 & 68.8102 \\
$3 / 52$ & 68.2401 & 68.2150 & 68.2379 & 68.2400 & 68.2400 \\
$4 / 52$ & 67.7622 & 67.7310 & 67.7612 & 67.7645 & 67.7644 \\
$1 / 12$ & 67.6155 & 67.5841 & 67.6168 & 67.6205 & 67.6204 \\
$5 / 52$ & 67.3340 & 67.3074 & 67.3449 & 67.3494 & 67.3494 \\
$6 / 52$ & 66.9237 & 66.9267 & 66.9715 & 66.9774 & 66.9773 \\
$7 / 52$ & 66.5095 & 66.5785 & 66.6305 & 66.6379 & 66.6378 \\
$8 / 52$ & 66.0811 & 66.2560 & 66.3152 & 66.3242 & 66.3241 \\
$1 / 6$ & 65.7871 & 66.0530 & 66.1168 & 66.1270 & 66.1268 \\
$9 / 52$ & 65.6382 & 65.9546 & 66.0208 & 66.0315 & 66.0314 \\
$10 / 52$ & 65.1857 & 65.6708 & 65.7440 & 65.7565 & 65.7563 \\
$11 / 52$ & 64.7297 & 65.4019 & 65.4822 & 65.4966 & 65.4963 \\
$12 / 52$ & 64.2757 & 65.1461 & 65.2333 & 65.2496 & 65.2494 \\
$1 / 4$ & 63.8278 & 64.9016 & 64.9958 & 65.0141 & 65.0138 \\
$14 / 52$ & 63.3889 & 64.6673 & 64.7683 & 64.7887 & 64.7884 \\
$15 / 52$ & 62.9608 & 64.4420 & 64.5498 & 64.5724 & 64.5720 \\
$16 / 52$ & 62.5446 & 64.2248 & 64.3395 & 64.3643 & 64.3638 \\
$17 / 52$ & 62.1408 & 64.0150 & 64.1365 & 64.1635 & 64.1630 \\
$1 / 3$ & 62.0090 & 63.9466 & 64.0703 & 64.0981 & 64.0976 \\
\hline
\end{tabular}

The "Highly Accurate" value is the converged numerical result obtained by numerically solving the following integral equation:

$$
\begin{aligned}
K-B_{t}^{p}= & K e^{-r(T-t)} N\left(-d_{2}\left(B_{t}^{p}, K, T-t\right)\right)-B_{t}^{p} e^{-q(T-t)} N\left(-d_{1}\left(B_{t}^{p}, K, T-t\right)\right) \\
& +\int_{t}^{T}\left[r K e^{-r s} N\left(-d_{2}\left(B_{t}^{p}, B_{s}^{p}, s\right)\right)-q B_{t}^{p} e^{-q s} N\left(-d_{1}\left(B_{t}^{p}, B_{s}^{p}, s\right)\right)\right] d s .
\end{aligned}
$$


Table 8: The price, $P(S, t)$, of an American put option with a strike price $K=100$ at time $t$ - Accuracy of the present truncated analytical formulas for the case $r<q$.

$$
\begin{aligned}
& P(S, t)=K e^{-r(T-t)} N\left(-d_{2}(S, K, T-t)\right)-S e^{-q(T-t)} N\left(-d_{1}(S, K, T-t)\right) \\
& +\int_{t}^{T}\left[r K e^{-r s} N\left(-d_{2}\left(S, B_{s}^{p}, s\right)\right)-q S e^{-q s} N\left(-d_{1}\left(S, B_{s}^{p}, s\right)\right)\right] d s, \\
& \text { where } B_{t}^{p}=\frac{r}{q} K e^{-2 \sqrt{\tau^{*}} w\left(\sqrt{\tau^{*}}\right)}, \quad \tau^{*}=\frac{1}{2} \sigma^{2}(T-t), \quad r^{*}=\frac{r}{\frac{1}{2} \sigma^{2}}, \quad q^{*}=\frac{q}{\frac{1}{2} \sigma^{2}}, \\
& \text { TA1 : } w\left(\sqrt{\tau^{*}}\right)=\beta_{0}, \quad \text { TA2 }: w\left(\sqrt{\tau^{*}}\right)=\beta_{0}+\beta_{1} \sqrt{\tau^{*}}, \\
& \text { TA3 : } w\left(\sqrt{\tau^{*}}\right)=\beta_{0}+\beta_{1} \sqrt{\tau^{*}}+\beta_{2} \tau^{*}, \quad \text { TA4 }: w\left(\sqrt{\tau^{*}}\right)=\beta_{0}+\beta_{1} \sqrt{\tau^{*}}+\beta_{2} \tau^{*}+\beta_{3} \tau^{* 3 / 2} \text {, } \\
& \beta_{0}=0.451723, \quad \beta_{1}=0.144914\left(r^{*}-q^{*}\right), \\
& \beta_{2}=-0.009801-0.041764\left(r^{*}+q^{*}\right)+0.014829\left(r^{*}-q^{*}\right)^{2} \text {, } \\
& \beta_{3}=-0.000618-0.002087\left(r^{*}-q^{*}\right)-0.015670\left(r^{* 2}-q^{* 2}\right)-0.001052\left(r^{*}-q^{*}\right)^{3} \text {. }
\end{aligned}
$$

Parameters: $r=0.05, q=0.07$ and $\tau \doteq T-t=1 / 12$

\begin{tabular}{|cccccc|}
\hline$(S, \sigma)$ & Highly Accurate & TA1 & TA2 & TA3 & TA4 \\
\hline$(90,0.2)$ & 10.1755 & 10.1755 & 10.1755 & 10.1755 & 10.1755 \\
$(95,0.2)$ & 5.6814 & 5.6814 & 5.6814 & 5.6814 & 5.6814 \\
$(100,0.2)$ & 2.3753 & 2.3754 & 2.3754 & 2.3754 & 2.3754 \\
$(105,0.2)$ & 0.6875 & 0.6875 & 0.6875 & 0.6875 & 0.6875 \\
$(110,0.2)$ & 0.1329 & 0.1329 & 0.1329 & 0.1329 & 0.1329 \\
$(90,0.3)$ & 10.5333 & 10.5333 & 10.5333 & 10.5333 & 10.5333 \\
$(95,0.3)$ & 6.5264 & 6.5263 & 6.5263 & 6.5263 & 6.5263 \\
$(100,0.3)$ & 3.5201 & 3.5202 & 3.5202 & 3.5202 & 3.5202 \\
$(105,0.3)$ & 1.6285 & 1.6285 & 1.6285 & 1.6285 & 1.6285 \\
$(110,0.3)$ & 0.6430 & 0.6430 & 0.6430 & 0.6430 & 0.6430 \\
$(90,0.4)$ & 11.1485 & 11.1485 & 11.1485 & 11.1485 & 11.1485 \\
$(95,0.4)$ & 7.4961 & 7.4961 & 7.4961 & 7.4961 & 7.4961 \\
$(100,0.4)$ & 4.6644 & 4.6645 & 4.6645 & 4.6645 & 4.6645 \\
$(105,0.4)$ & 2.6781 & 2.6781 & 2.6781 & 2.6781 & 2.6781 \\
$(110,0.4)$ & 1.4193 & 1.4193 & 1.4193 & 1.4193 & 1.4193 \\
$(90,0.5)$ & 11.9189 & 11.9188 & 11.9188 & 11.9188 & 11.9188 \\
$(95,0.5)$ & 8.5209 & 8.5209 & 8.5209 & 8.5209 & 8.5209 \\
$(100,0.5)$ & 5.8077 & 5.8079 & 5.8079 & 5.8079 & 5.8079 \\
$(105,0.5)$ & 3.7743 & 3.7744 & 3.7744 & 3.7744 & 3.7744 \\
$(110,0.5)$ & 2.3416 & 2.3417 & 2.3417 & 2.3417 & 2.3417 \\
\hline RMSE & & 0.0001 & 0.0001 & 0.0001 & 0.0001 \\
MAE & & 0.0002 & 0.0002 & 0.0002 & 0.0002 \\
\hline
\end{tabular}

The "Highly Accurate" value is obtained by numerically computing the integration with the highly accurate critical stock prices presented in Table 7 . RMSE is the root of the mean squared errors. MAE is the maximum absolute error. 
Table 9: The price, $P(S, t)$, of an American put option with a strike price $K=100$ at time $t$ - A comparison between three formulas (19), (20) and (21) for $|r-q| \ll 1$.

Parameters: $r=0.05$ and $\tau \doteq T-t=1 / 12$

\begin{tabular}{|ccccc|}
\hline$(S, \sigma)$ & Highly Accurate & Formula1 & Formula2 & Formula3 \\
\hline$(90,0.2)$ & 10.0535 & 10.0440 & 10.0543 & 10.0731 \\
$(95,0.2)$ & 5.5603 & 5.5573 & 5.5604 & 5.5746 \\
$(100,0.2)$ & 2.2947 & 2.2939 & 2.2948 & 2.2986 \\
$(105,0.2)$ & 0.6537 & 0.6535 & 0.6537 & 0.6545 \\
$(110,0.2)$ & 0.1242 & 0.1241 & 0.1242 & 0.1243 \\
$(90,0.3)$ & 10.4167 & 10.4099 & 10.4172 & 10.4432 \\
$(95,0.3)$ & 6.4202 & 6.4173 & 6.4203 & 6.4349 \\
$(100,0.3)$ & 3.4415 & 3.4404 & 3.4416 & 3.4473 \\
$(105,0.3)$ & 1.5812 & 1.5807 & 1.5812 & 1.5832 \\
$(110,0.3)$ & 0.6197 & 0.6196 & 0.6198 & 0.6204 \\
$(90,0.4)$ & 11.0410 & 11.0350 & 11.0413 & 11.0696 \\
$(95,0.4)$ & 7.3994 & 7.3964 & 7.3995 & 7.4150 \\
$(100,0.4)$ & 4.5875 & 4.5862 & 4.5877 & 4.5952 \\
$(105,0.4)$ & 2.6239 & 2.6232 & 2.6239 & 2.6274 \\
$(110,0.4)$ & 1.3851 & 1.3847 & 1.3850 & 1.3865 \\
$(90,0.5)$ & 11.8191 & 11.8134 & 11.8194 & 11.8484 \\
$(95,0.5)$ & 8.4307 & 8.4274 & 8.4308 & 8.4475 \\
$(100,0.5)$ & 5.7326 & 5.7310 & 5.7329 & 5.7422 \\
$(105,0.5)$ & 3.7164 & 3.7154 & 3.7165 & 3.7215 \\
$(110,0.5)$ & 2.2998 & 2.2994 & 2.3000 & 2.3026 \\
\hline
\end{tabular}

The "Highly Accurate" value is obtained by numerically computing the integration with the highly accurate critical stock prices presented in Table 5 for $q=0.05$. The "Formula1" value is obtained by using formula (19) for the region $r>q \geq 0$ with $q=0.0499$. The "Formula2" value is obtained by using formula (20) for the region $r=q$ with $q=0.05$. The "Formula3" value is obtained by using formula (21) for the region $r<q$ with $q=0.0501$. 